\title{
Scaling of the streamwise velocity component in turbulent pipe flow
}

\author{
By J. F. MORRISON ${ }^{1}$, B. J. McKEON ${ }^{2}$, W. JIANG ${ }^{3}$ \\ AND A. J. SMIT S ${ }^{2}$ \\ ${ }^{1}$ Department of Aeronautics, Imperial College, London SW7 2AZ, UK \\ ${ }^{2}$ Department of Mechanical \& Aerospace Engineering, Princeton University, \\ Princeton, NJ 08544-0710, USA \\ ${ }^{3}$ CARDC, PO Box 211 Mianyang, Sichuan 621 000, People's Republic of China
}

(Received 7 March 2003 and in revised form 30 January 2004)

Statistics of the streamwise velocity component in fully developed pipe flow are examined for Reynolds numbers in the range $5.5 \times 10^{4} \leqslant R e_{D} \leqslant 5.7 \times 10^{6}$. Probability density functions and their moments (up to sixth order) are presented and their scaling with Reynolds number is assessed. The second moment exhibits two maxima: the one in the viscous sublayer is Reynolds-number dependent while the other, near the lower edge of the log region, follows approximately the peak in Reynolds shear stress. Its locus has an approximate $\left(R^{+}\right)^{0.5}$ dependence. This peak shows no sign of 'saturation', increasing indefinitely with Reynolds number. Scalings of the moments with wall friction velocity and $\left(U_{c l}-\bar{U}\right)$ are examined and the latter is shown to be a better velocity scale for the outer region, $y / R>0.35$, but in two distinct Reynoldsnumber ranges, one when $R e_{D}<6 \times 10^{4}$, the other when $R e_{D}>7 \times 10^{4}$. Probability density functions do not show any universal behaviour, their higher moments showing small variations with distance from the wall outside the viscous sublayer. They are most nearly Gaussian in the overlap region. Their departures from Gaussian are assessed by examining the behaviour of the higher moments as functions of the lower ones. Spectra and the second moment are compared with empirical and theoretical scaling laws and some anomalies are apparent. In particular, even at the highest Reynolds number, the spectrum does not show a self-similar range of wavenumbers in which the spectral density is proportional to the inverse streamwise wavenumber. Thus such a range does not attract any special significance and does not involve a universal constant.

\section{Introduction}

It has long been accepted that the motion in the viscous sublayer $\left(y^{+}=y u_{\tau} / \nu<30\right.$, where $u_{\tau}=\sqrt{\tau_{w} / \rho}, y$ is the wall-normal distance and $\tau_{w}$ is the wall shear stress) is directly affected by viscosity, $v$. Either a conventional overlap analysis that uses asymptotic matching (Millikan 1938; Wosnik, Castillo \& George 2000) or a dimensional analysis that yields the logarithmic law for the mean velocity suggests that the motion of the log region is independent of viscosity. However, such analyses do not specify directly the range in $y$ over which the log law applies, this being the subject of much experimental investigation. Zagarola \& Smits (1998) have shown that the mean velocity exhibits a log-law dependence for $600<y^{+}<0.07 R^{+}$only when $R^{+}>9 \times 10^{3}\left(R^{+}\right.$is the Kármán number based on pipe radius) and that 
for $60<y^{+}<500$, there is a power law when $R^{+}>9000$ which expresses the direct influence of viscosity out to $y^{+} \approx 0.15 R^{+}$when $R^{+}<9000$ (see also Zagarola, Perry $\&$ Smits 1997). Using an improved data analysis, McKeon et al. (2004) have recently confirmed that complete similarity in the form of a log law (with slightly modified constants) occurs for $R^{+}>5 \times 10^{3}$ only, in the range $600<y^{+}<0.12 R^{+}$. These scalings for the first moment therefore raise questions concerning the most appropriate choice of velocity scale for the higher moments, particularly because the fluctuating motion at a point comprises a range of scales, or equivalently, motion at a given wavenumber receives contributions from the entire physical domain. Moreover, near walls much of the turbulence information resides in the smaller scales which, in addition to inhomogeneity and anisotropy, show significant departures from a Gaussian velocity distribution. For example, close to the wall, the flatness of the wall-normal velocity component becomes very large (Eggels et al. 1994) and can be attributed to the spatially alternating behaviour of ejections and sweeps. Statistically, this suggests inter-connection between many possible degrees of freedom, so contravening one of the requirements of the central limit theorem. The behaviour of the higher moments at very high Reynolds numbers is therefore very interesting.

In contrast to the mean velocity, it has been established for some time that the Reynolds stresses near the wall scale neither with 'wall' (or 'inner') variables $\left(v / u_{\tau}, u_{\tau}\right)$, nor with 'outer' variables $\left(R, u_{o}\right.$, where $u_{o}$ is an outer velocity scale left undefined at present). Of particular importance is the difference between the behaviour of the wall-parallel $(u, w)$ and wall-normal $(v)$ components where the impermeability constraint, which affects eddies out to a distance from the wall that is of the order of the eddy size ('blocking' or 'splatting'), is responsible for an increase in the wallparallel components at the expense of the wall-normal one. Thus the behaviour of the statistics for the $u$ - and $w$-components is different from those for the $v$-component, and in the case of pipe flow, the streamwise and azimuthal components are subject to specialized homogeneous boundary conditions. Failure of the $u$ - and $w$-components to scale on wall variables was explained by Townsend $(1961,1976)$ as the influence of 'inactive' motion (Bradshaw 1967; Morrison, Subramanian \& Bradshaw 1992), that of the large eddies inducing a 'meandering or swirling' on the near-wall motion. Being largely confined to the $(x, z)$-plane and scaling on outer variables, the inactive motion does not, to a first order, contribute to either the $\rho \overline{v^{2}}$ normal stress, or to the shear stress, $-\rho \overline{u v}$, the 'active' component of near-wall motion. It is therefore supposed that the two modes do not interact, the active one being modulated by an irrotational free stream' (Bradshaw 1967), the result of both large-scale vorticity and irrotational pressure fluctuations.

For 'high' Reynolds numbers, when $y^{+} \gg 1$ and $y / R \ll 1$, an overlap region for the first moment only of the streamwise component becomes apparent. Even then, there are many measurements that show that the active component of the Reynolds stresses does not scale on inner variables either. In particular, the 'constant-stress' region $\left(-\overline{u v} \approx u_{\tau}^{2}\right)$ does not hold, except in the limit of very high Reynolds number. But it is by no means clear just how high a Reynolds number is required: this is particularly noteworthy since the constant-stress region can be deduced by the same dimensional arguments that lead to the log law, but it has yet to be determined whether or not the constant-stress region emerges at about the same Reynolds number as that at which the log law does (Zagarola \& Smits 1998; McKeon et al. 2004). Morrison et al. (1992) show that the Reynolds-stress-bearing motion in a boundary layer at $\operatorname{Re}_{\theta} \approx 1.5 \times 10^{4}$ (equivalent to $R^{+} \approx 5000$ ) is associated with a Kolmogorov scaling and this should be recognized as evidence of the failure of universal inner 
scaling, that is, $u_{\tau}=-(\overline{u v})^{1 / 2}$ with lengthscale $y, y^{+} \gg 1$. See also Antonia \& Kim (1994) and Wei \& Willmarth (1989). These studies were performed at much lower Reynolds numbers than those studied in the present investigation.

Nevertheless, in considering the Reynolds-number dependence of near-wall turbulence, it is important to distinguish direct viscous influence (physically, the eruptions of low-momentum fluid from the sublayer) from that of the outer-region motion (large-scale inrushes that produce splats near the wall). The ratio of the two relevant lengthscales is, of course, $R^{+}$. Not only is the outer-scaling influence of inactive motion more apparent at high Reynolds numbers, it is also more prevalent in boundary layers in which the influence of inactive motion is larger than in internal flows. This raises the question of the extent to which the active and inactive modes interact, and indeed whether such a delineation is meaningful. In this context, it is important to remember that Townsend's original distinction was based on the basic, but conceptual element of near-wall structure, the 'attached wall eddy'. Recently, the precise nature of so-called inactive motion in high-Reynolds-number boundary layers has been examined by Hunt \& Morrison (2000) who suggest that 'top-down', outerlayer interactions are important to the dynamics in the near-wall region. Note that any interaction between these two modes implies that, even at very high Reynolds numbers when direct viscous effects are small, wall motion cannot be universal in any meaningful sense. Outer-layer influences may also have ramifications for the self-similarity of the mean velocity: both Bradshaw (1967) and Townsend (1976) use a simple linear analysis to show that the effect of inactive motion is to make the von Kármán constant, $\kappa$, Reynolds-number dependent. These considerations lead also to the conclusion that simple arguments concerning the overlap of scales for the higher moments in a particular region of wall turbulence are inappropriate. Crucial to the understanding of these issues is the realization that the wall affects wall-normal and wall-parallel components differently.

In this paper, we report hot-wire measurements of the streamwise velocity component in the Reynolds-number range (based on pipe diameter, $D$ and mean velocity, $\bar{U}) 5.5 \times 10^{4} \leqslant R e_{D} \leqslant 5.7 \times 10^{6}$. Statistics up to the sixth moment are calculated as the moments of a probability density function (p.d.f.). Equivalent spectra as a function of streamwise wavenumber, $\phi\left(k_{1}\right)$, are also presented. The scaling of both is investigated. It is becoming increasingly apparent that there are significant differences between different flows of the same species: thus in the present context, we distinguish between not only external and internal flows, but also between pipe and channel flows (Nieuwstadt \& Bradshaw 1997). Therefore reference to channel flows is not made unless the results are specifically relevant to the present work. Early work on pipe flow includes that of Laufer (1954) and Sandborn (1955) and more recently, Durst, Jovanovic \& Sender (1995), Eggels et al. (1994) and Fontaine \& Deutsch (1995). However, all of these studies are limited in terms of the range of Reynolds numbers over which data were obtained. The main influence, pervading much of the work reported here, comes from Townsend's seminal work concerning the self-similar structure of attached wall eddies, which forms the basis of the supposed self-similarity of the spectra for the surface-parallel velocities and the functional forms for the normal stresses. This stimulated further work, principally that of Perry \& Abell (1975, 1977) and Perry, Henbest \& Chong (1986) (pipes) and Perry \& Li (1990), Perry \& Marusic (1995), Marusic \& Perry (1995), Marusic, Uddin \& Perry (1997) and Jones, Marusic \& Perry (2001) (boundary layers). Marusic \& Kunkel (2003) have recently extended these ideas. In later sections, we interpret the data using the concept of inactive motion. But, owing to the very significant potential benefits accruing from a 
self-similar description of wall turbulence, it seems prudent first to examine the exact requirements for this to be so.

\section{Similarity considerations}

Zagarola \& Smits (1998) show that asymptotic matching of the mean velocity gradients in the overlap region leads to complete similarity (in the form of the log law) for $600<y^{+}<0.07 R^{+}$when the Reynolds number is sufficiently high and when the velocity scales for the inner and outer regions are the same, that is, given by $u_{\tau}$. (See also Zagarola et al. 1997; McKeon, Li, Jiang, Morrison \& Smits 2004) At smaller $y^{+}$, they argue that the ratio of inner to outer velocity scales, $u_{\tau} / u_{o}$, is a function of $R^{+}$, and that simultaneous matching of both the velocities and velocity gradients leads to a power law. The appearance of a power law may be regarded as a form of incomplete similarity and is supported by the data of both Zagarola \& Smits (1998) for $60<y^{+}<500$ and McKeon et al. (2004) for $60<y^{+}<300$. The term 'complete (or self-) similarity' means that, first, the lengthscale used to normalize the independent variable, $y$, in the log argument may be freely chosen, and second, the von Kármán constant is universal. For this reason the log law is valid using inner or outer scaling, or even using a rough-surface lengthscale. In fact, demonstration of complete similarity requires simultaneous collapse using both inner and outer scaling.

Owing to the difficulties in scaling the Reynolds stresses near the wall, there have been several attempts at finding a more suitable inner velocity scale. Prominent among the alternatives to $u_{\tau}$ is so-called 'mixed' scaling (where the velocity scale is $\left(u_{\tau} U_{c l}\right)^{1 / 2}$, see for example, DeGraaff \& Eaton 2000) for the horizontal stresses. Zagarola \& Smits (1998) have suggested that a true outer velocity scale is $U_{c l}-\bar{U}$, where $U_{c l}$ is the centreline velocity. For $R e_{D}>2 \times 10^{5}$, they show that $\left(U_{c l}-\bar{U}\right) / u_{\tau} \longrightarrow 4.34$ although, with more data and a revised analysis, McKeon et al. (2004) suggest that the constant is 4.28 . The use of both mixed scaling and $\left(U_{c l}-\bar{U}\right)$ as a velocity scale is considered in $\S 5$.

Since publication of Townsend's seminal work, considerable attention has been devoted to the deduction of spectral forms associated with the self-similar nature of attached wall eddies. Such self-similarity manifests itself at 'high' Reynolds numbers as a range of streamwise wavenumber, $k_{1}$, in which the spectrum $\phi_{11} \propto u_{\tau}^{2} k_{1}^{-1}$. There are several derivations, the earliest provided by Tchen (1953), reappraised by Hinze (1975), involving the balance between the spectral transfer of energy by the mean shear and that by inertial interactions of the turbulence - a strong interaction or 'resonance' condition. Tchen's theory involves several assumptions that are questionable in highly anisotropic wall turbulence, such as a constant strain rate and a spherically symmetric eddy viscosity. As such, the individual components are not distinguished. More pragmatically, it should be noted that a prescribed slope over some region of wavenumber can usually be found in turbulence spectra on log-log axes. The simpler theory for pipe flow was proposed by Perry \& Abell (1977) and Perry et al. (1986), but it is equally appropriate for boundary layers (see papers by Perry and colleagues). The theory has been the subject of much attention, but it appears that often the existence of self-similarity at practical Reynolds numbers is taken for granted (Nikora 1999; Högström, Hunt \& Smedman 2002), or that its proof is the result of $a b$ initio assumptions (Kader \& Yaglom 1991). Given the prominence of the theory and, in terms of the Reynolds number, the uniqueness of the present data, a careful reappraisal is clearly needed (see also Morrison et al. 2002a, b). In this, 
it would appear sensible to focus on that range of $y$ in which the mean velocity is known to exhibit self-similarity in the form of a log law.

'Large' scales (in which the direct effects of viscosity may be neglected) that contribute to the streamwise velocity component may be scaled using either inner or outer scales. Outer scaling suggests that $y$ is not important and, taking $u_{\tau}$ as the appropriate velocity scale, dimensional analysis therefore yields

$$
\frac{\phi_{11}\left(k_{1}\right)}{R u_{\tau}^{2}}=\frac{\phi_{11}\left(k_{1} R\right)}{u_{\tau}^{2}}=g_{1}\left(k_{1} R\right),
$$

while, alternatively, inner scaling suggests the exclusion of $R$ as a relevant lengthscale so that, at higher wavenumbers,

$$
\frac{\phi_{11}\left(k_{1}\right)}{y u_{\tau}^{2}}=\frac{\phi_{11}\left(k_{1} y\right)}{u_{\tau}^{2}}=g_{2}\left(k_{1} y\right) .
$$

The veracity of these scalings is usually judged by the degree of collapse of the spectra at wavenumbers lower than that at which spectral transfer (which at high Reynolds numbers is given by the mean dissipation rate) becomes important. In the range of wave numbers $R^{-1}<k_{1}<y^{-1}$ over which both (2.1) and (2.2) are valid (that is collapse is evident with both scalings, as required by asymptotic matching), it then follows that

$$
\phi_{11}\left(k_{1}\right)=R u_{\tau}^{2} g_{1}\left(k_{1} R\right)=y u_{\tau}^{2} g_{2}\left(k_{1} y\right) .
$$

Dimensional arguments and direct proportionality between $g_{1}$ and $g_{2}$ therefore imply

$$
\frac{\phi_{11}\left(k_{1} R\right)}{u_{\tau}^{2}}=\frac{A_{1}}{k_{1} R}=g_{1}\left(k_{1} R\right),
$$

and

$$
\frac{\phi_{11}\left(k_{1} y\right)}{u_{\tau}^{2}}=\frac{A_{1}}{k_{1} y}=g_{2}\left(k_{1} y\right),
$$

where $A_{1}$ is a universal constant. Collapse with both length scales therefore suggests a self-similar structure such that $\phi_{11}\left(k_{1}\right) \propto u_{\tau}^{2} k_{1}^{-1}$. We will therefore call this situation 'complete similarity'. In this situation, the only relevant lengthscale is $k_{1}^{-1}$ itself, and, owing to the nature of the Fourier transform and because the foregoing analysis is equally valid for the spanwise velocity component, a self-similar structure would have to be space-filling in $(x, z)$-planes parallel to the surface. Now, it is possible that, for example, while $y$ and $u_{\tau}$ might form a complete parameter set to define the motion in the range of wavenumbers over which collapse is apparent with (2.2), these wave numbers might, in fact, be too high for collapse to be possible using $R$ and $u_{\tau}$ as in (2.1). Thus simultaneous collapse is not possible. We shall refer to this situation as 'incomplete similarity', in which case the constant $A_{1}$ in (2.4) and (2.5) cannot be universal.

Note that this analysis is predicated on two principal assumptions. The first is that the kinematic viscosity, $v$, does not enter the problem. This requires that $k_{1} v / u_{\tau} \ll 1$. In turn, this requires the Reynolds number to be sufficiently high, or equivalently that $y$ is sufficiently large, such the energy-containing scales are not affected directly by viscosity. Taking the outer limit to the power-law region for the first moment to be $y^{+}=500$, it would seem unlikely that higher moments would be free of direct viscous effects below $y^{+} \approx 1000$, as shown by the conditional sampling results of Morrison et al. (1992). The second assumption is that $u_{\tau}$ is the correct velocity scale for both the inner and outer regions. In particular, in conformity with Townsend's theory, 
it supposes that inactive motion arises primarily through the influence of attached eddies and that therefore $u_{\tau}$ is the appropriate velocity scale. The analysis does not specify $u_{\tau}$ to be the velocity scale: rather, it specifies that the velocity scale should be the same with both inner and outer scaling, without which complete similarity would not be possible. Note also that this analysis does not apply to the wall-normal velocity component which is blocked at wavenumbers, $k_{1} \sim y^{-1}$.

In $\S 4$, spectra are presented in premultiplied form on linear-log axes. A linear ordinate enables a closer scrutiny of scalings than that afforded by a logarithmic one. In addition, the use of non-dimensional axes ensures that not only the ordinate but also the area under the spectra is directly proportional to energy. Integration of the spectra yields ${\overline{u^{2}}}^{+}=\overline{u^{2}} / u_{\tau}^{2}$. Spectra are therefore in the form

$$
\frac{k_{1} R \phi_{11}\left(k_{1} R\right)}{u_{\tau}^{2}}=h_{1}\left(k_{1} R\right)
$$

for outer scaling, and in the form

$$
\frac{k_{1} y \phi_{11}\left(k_{1} y\right)}{u_{\tau}^{2}}=h_{2}\left(k_{1} y\right)
$$

for inner scaling. In the context of assessing these scalings for data in the present experiment, it is useful to clarify precisely what the foregoing analysis indicates. Strictly, as long as $v / u_{\tau} \ll y \ll R$ (the Reynolds number is 'high'), (2.4) and (2.5) should both show a $k_{1}^{-1}$ range for $R^{-1} \ll k_{1} \ll y^{-1}$. However, in order to remove the ambiguity concerning the relative values of $y$ and $R$, one alternatively may fix $y$ in (2.4) and then $R$ in (2.5). Equation (2.6) invites us to retain only $R$ and $u_{\tau}$ as independent variables. Thus while $y$ is fixed, $u_{\tau}$ is varied by changing the pressure drop along the pipe. In practice, this involves a change of Reynolds number (strictly Kármán number) as changes of $R$ are a little more problematical. This does not pose a problem as long as the Reynolds number is sufficiently high such that the wave number range of interest is not directly affected by viscosity. Alternatively, (2.7) invites the use of $y$ and $u_{\tau}$ only as independent variables for any fixed $R$. In this case, $y$ can merely be varied (subject to $v / u_{\tau} \ll y \ll R$ ) at a fixed Reynolds number, although as long as $v$ can be neglected, a value of $y$ at any Reynolds number might be chosen. For brevity, we present spectra (obviously using both inner and outer scaling) at different $y / R$ for the lowest and highest Reynolds number. The self-similarity (or not) of the $k_{1}^{-1}$ range is discussed in the light of the present results in $\S 5$.

Accepting $u_{\tau}$ as a velocity scale for both inner and outer regions makes possible an overlap analysis with $y$ (as well as $k_{1}$ ) as the dependent variable. The use of $u_{\tau}$ is almost universal (e.g. see Perry \& Abell 1977). Wosnik et al. (2000) suggest that this has to be

$$
u_{\tau}=\sqrt{-\frac{R}{\rho} \frac{\mathrm{d} P_{0}}{\mathrm{~d} x}}=\sqrt{-\frac{R}{\rho} \frac{\mathrm{d} P}{\mathrm{~d} x}},
$$

as defined by the momentum equation in the outer layer. Note however that this assumes that the viscous term is negligible and that streamwise homogeneity of $\overline{v^{2}}$ leads to equality of the static-pressure and total-pressure gradients. Their analysis for the overlap region leads to a logarithmic dependence for all the Reynolds stresses of the form

$$
{\overline{u^{2}}}^{+}=A\left[R^{+}\right] \ln \left[y^{+}+a^{+}\right]+B\left[R^{+}\right]
$$


where $A$ and $B$ are functions of Reynolds number that are asymptotically constant. The offset is given by $0 \leqslant a^{+} \leqslant-16$ and is required to account for the existence of a 'mesolayer' (Long \& Chen 1981). However, the validity of equation (2.8) rests upon the scaling of the Reynolds stresses being the same as that for the mean velocity in the overlap region (hence the log dependence). But it is clear from the foregoing arguments concerning active and inactive motion that first - as we shall show - there is no simple scaling between the Reynolds stresses and the mean velocity and, second, the behaviour of the individual components of the stress tensor depends crucially on the velocity components involved. It is always useful to remember that the first moment of a turbulence quantity says nothing about the higher moments: more physically, this means simply that the frame-dependent mean velocity is never a scale for the turbulence. These considerations are also relevant to the appropriateness of mixed scaling.

Based on considerations of the self-similar structure of attached wall eddies, Townsend (1976), Perry \& Abell (1977) and Perry et al. (1986) have suggested other logarithmic functional forms for the normal stresses of the surface-parallel velocities:

$$
{\overline{u^{2}}}^{+}=B_{1}-A_{1} \ln \left[\frac{y}{R}\right]-C\left(y^{+}\right)^{-0.5} .
$$

While (2.9) has a similar functional form to that of (2.8), the log term is obtained by integrating (2.4) or (2.5) for $R^{-1}<k_{1}<y^{-1}$. For comparison with the present data, we take the constants suggested by Perry, Henbest \& Chong (1986): $B_{1}=2.67, A_{1}=0.9$ and $C=6.06$. Previously, Perry \& Abell (1977) used constants $B_{1}=3.53, A_{1}=0.8$ and $C=9.54$, but this makes no difference to our conclusions concerning the proposed functional form. Marusic et al. (1997) extended (2.9) to include a wake deviation term, $W_{g}$, appropriate for boundary layers:

$$
\frac{\overline{u^{2}}}{u_{\tau}^{2}}=B_{1}-A_{1} \ln \left[\frac{y}{R}\right]-V_{g}\left[y^{+}\right]-W_{g}\left[\frac{y}{R}\right],
$$

which also has a $\log$ dependence. $V_{g}\left[y^{+}\right]$is a viscous deviation term operative at small $y^{+}$, while $W_{g}$ is effective at large $y / R$. In $\S 4$ we compare both (2.8) and (2.9) with the present data.

\section{Experimental techniques}

Measurements are made in a closed-loop, compressed-air facility, in which an extruded aluminium pipe with nominal diameter of $129 \mathrm{~mm}$ is mounted. The facility is described in detail by Zagarola \& Smits (1998), with particular attention paid to assembly, alignment and surface finish of the test pipe. Air is driven by an impeller mounted in a pumping section which is followed by a heat exchanger, the return leg, a flow-conditioning section and a test section beginning at $160 \mathrm{D}$ downstream of the contraction exit, with all measurements made at $164 D$. By comparison, the intensity and spectral measurements of Perry \& Abell $(1975,1977)$ at $R e_{D}=3.0 \times 10^{5}$, for example, were performed at 71.9D and 86.2D downstream of the tripping device. Dean \& Bradshaw (1976) show that for channel flow, profiles of flatness of the streamwise velocity fluctuation are independent of development length after 67 channel heights. Zagarola \& Smits (1998) estimate that the development length increases from $78 D$ to $131 D$ for an increase of Reynolds number from $R e_{D}=3.0 \times 10^{5}$ to $4.0 \times 10^{7}$. Therefore, in the case of the present measurements of the streamwise velocity 


$\begin{array}{cccccc}R e_{D} & R^{+} & l / d & l^{+} & \begin{array}{c}\text { Spatial resolution } \\ k_{1} \eta\end{array} & \begin{array}{c}\text { Averaging time } \\ T U_{c l} / R\end{array} \\ 5.5 \times 10^{4} & 1.50 \times 10^{3} & 200 & 11.6 & 1.08 & 2.20 \times 10^{5} \\ 7.5 \times 10^{4} & 1.82 \times 10^{3} & 200 & 14.1 & 0.89 & 3.26 \times 10^{4} \\ 1.5 \times 10^{5} & 3.35 \times 10^{3} & 200 & 25.9 & 0.48 & 3.20 \times 10^{4} \\ 2.3 \times 10^{5} & 5.08 \times 10^{3} & 200 & 39.4 & 0.32 & 8.88 \times 10^{3} \\ 4.1 \times 10^{5} & 8.56 \times 10^{3} & 100 & 33.1 & 0.41 & 2.13 \times 10^{4} \\ 1.0 \times 10^{6} & 1.97 \times 10^{4} & 100 & 76.0 & 0.21 & 8.45 \times 10^{3} \\ 3.1 \times 10^{6} & 5.52 \times 10^{4} & 100 & 213.0 & 0.092 & 1.14 \times 10^{4} \\ 5.7 \times 10^{6} & 1.01 \times 10^{5} & 100 & 385.0 & 0.059 & 2.52 \times 10^{5}\end{array}$

TABLE 1. Principal parameters of data sets.

component for Reynolds numbers $5.5 \times 10^{4} \leqslant R e_{D} \leqslant 5.7 \times 10^{6}$, we take moments up to sixth order to be independent of streamwise location.

Measurements are made using hot wires with variable length-to-diameter ratios, $l / d$, and etched using standard techniques. All data sets were obtained using $2.5 \mu \mathrm{m}$ diameter, platinum/rhodium wire except those at $R e_{D}=4.1 \times 10^{5}$ and $1.0 \times 10^{6}$ which were obtained using tungsten wire of the same diameter. Values of $l / d$ used for these data sets are shown in table 1, along with all other relevant parameters. Hot-wire calibration is performed using a fourth-order polynomial, the signal being sampled using 12-bit A-D conversion. A maximum permissible change of $1 \%$ in mean velocity is required between calibrations taken either before and after traverses, or before and after long sampling periods (for spectra). The wall shear stress used for scaling purposes is calculated from contemporary measurements of the pressure drop as described by Zagarola \& Smits (1998).

Difficulties that relate particularly to the use of hot wires at high Reynolds numbers are dealt with in detail by Li et al. (2004). A principal difficulty is the choice of a minimum $l / d$ that provides a sufficiently uniform wire temperature distribution in spite of heat conduction to the supporting stubs and prongs. Owing to the increased forced convection from the wire as the Reynolds number increases, $l / d$ may be reduced without increasing the fractional heat loss through wire-stub conduction. Using a static solution of the heat-balance equation for both the wire and stubs, Li et al. (2004) calculate the heat loss by conduction for a range of wire materials, $\mathrm{stub}$ /wire length ratios and wire Reynolds numbers, $R e_{w}$. Following Freymuth (1979), a static fractional heat loss due to conduction, $K$, may be defined as

$$
\sigma=\frac{K}{K+\psi}
$$

where $\psi$ is the heat loss due to forced convection. An equivalent quantity for the dynamic fractional heat loss, $\sigma^{\prime}$, may also be defined where $K^{\prime}$ and $\psi^{\prime}$ represent, respectively, the first derivative of $K$ and $\psi$ with respect to wire Biot number. The calculations for platinum wire with an overheat ratio, $a=0.82$ at $R e_{w}=3.2$ show that, for wires with $l / d \approx 200$ and short stubs, $\sigma=7 \%$, although precise values depend on the choice of wire material and stub length. However, the calculations do confirm the measured relative conduction end losses of Champagne, Sleicher \& Wehrmann (1967) and we have therefore taken $\sigma \leqslant 7 \%$ as the principal criterion governing the minimum permissible $l / d$ for wires up to $R e_{w} \approx 250$. 
For comparison, Li et al. (2004) also use Freymuth's solution for (3.1) (see also Corrsin 1963) that ignores heat generation within and convection from the stubs. This requires the assumption that the wire-end temperature is that of the wire-stub junction, while the Freymuth 'conduction-only' model takes the wire-end temperature to be the ambient temperature. The agreement is very good, except when $l / d$ is so small $(<100)$ and $K$ is large so that the model is very sensitive to the choice of wireend temperature. This agreement may be used as a justification for making estimates of $\sigma^{\prime}$ using the conduction-only model. This has also been shown to be accurate by Morris \& Foss (2003) who use a time-dependent thermal model of a hot wire with a modelled ideal feedback amplifier to estimate $\sigma^{\prime}$.

Table 1 shows that, at the lowest Reynolds number, data are taken with a hot wire for which $l / d=200$. In this case, $R e_{w} \approx 3$ and therefore the criterion $\sigma<7 \%$ is met. Table 1 further shows that end-conduction losses are most critical for the data set $R e_{D}=4.1 \times 10^{5}$, the lowest Reynolds number at which wires with $l / d=100$ are used. For these data, $3.7 \leqslant R e_{w} \leqslant 11.2$. The calculations of Li et al. (2004) for a platinum wire suggest that, in order to meet the criterion of $\sigma<7 \%, l / d \geqslant 145$ at $R e_{w}=3.7$. However, the conduction losses are increased by the use of, for this data set, tungsten wire for which the thermal conductivity is about twice that of platinum. Yet, all the hot wires used here had much longer stub lengths than those for which calculations were performed. The calculations therefore suggest a worst-case estimate of $\sigma \approx 15 \%$ for the data point closest to the wall.

Of more importance are the estimates of $\sigma$ and $\sigma^{\prime}$ for the data taken at the highest Reynolds number, $R e_{D}=5.7 \times 10^{6}$, and for which $R e_{w} \approx 250$. Here, calculations indicate that for platinum wire, $l / d$ may be reduced to about 50 , although precise limits depend on the value of $R e_{w}$. Use of the conduction-only model for a platinum wire of length $l / d=100$ operated with $a=0.82$ and $R e_{w}=250$ gives $\sigma=2.3 \%$ and $\sigma^{\prime}=0.84 \%$. Therefore, end-conduction effects at the highest Reynolds number are significantly less than those for wires with $l / d=200$ at $R e_{w} \approx 3$. Li et al. (2004) provide full details. Other less critical issues are related to the bridge frequency response. These include the generation of heat waves along the wire and bridge stability at high output voltages. These are also dealt with by Li et al. (2004).

All statistics are calculated as moments of probability density functions (p.d.f.). Spectra are calculated using, typically, data records of $1800 \mathrm{~s}$ duration. Table 1 shows the principal parameters governing the flow conditions as well as details concerning the spatial and temporal resolution of the data. The spatial resolution is expressed non-dimensionally as $l^{+}=l u_{\tau} / v$ as well as $k_{1} \eta$, where $\eta$ is the Kolmogorov lengthscale and $k_{1} l=2 \pi$, which is independent of the pipe flow conditions; $\eta$ is deduced from the local-equilibrium approximation of the dissipation rate, $\epsilon$, at mid $\log$ region and is typically $10 \%$ larger than that deduced from the third-order structure function. The sampling frequency was varied between $20 \mathrm{kHz}$ and $100 \mathrm{kHz}$ and was set so that the Nyquist frequency expressed as a wavenumber, $k_{1}=2 \pi f / U$, exceeds that equivalent to the limit of spatial resolution for the worst case situation of data at the pipe centreline. Typically, the bridge frequency response was $60-75 \mathrm{kHz}$ with $a \approx 0.8$. The signal was low-pass filtered at the Nyquist frequency and standard FFT algorithms (Hanning window) are used to calculate the spectra as a function of $k_{1}$.

Convergence of p.d.f. moments is better than $1 \%$, except in the case of the third and fifth moments in the region $10 \leqslant y^{+} \leqslant 30$ where it is better than $10 \%$ only. This increased error can plausibly be attributed to flow behaviour which induces large changes of both magnitude and sign. The fact that the fourth and sixth moments have smaller errors can be explained by the nonlinear behaviour of all anemometers. 


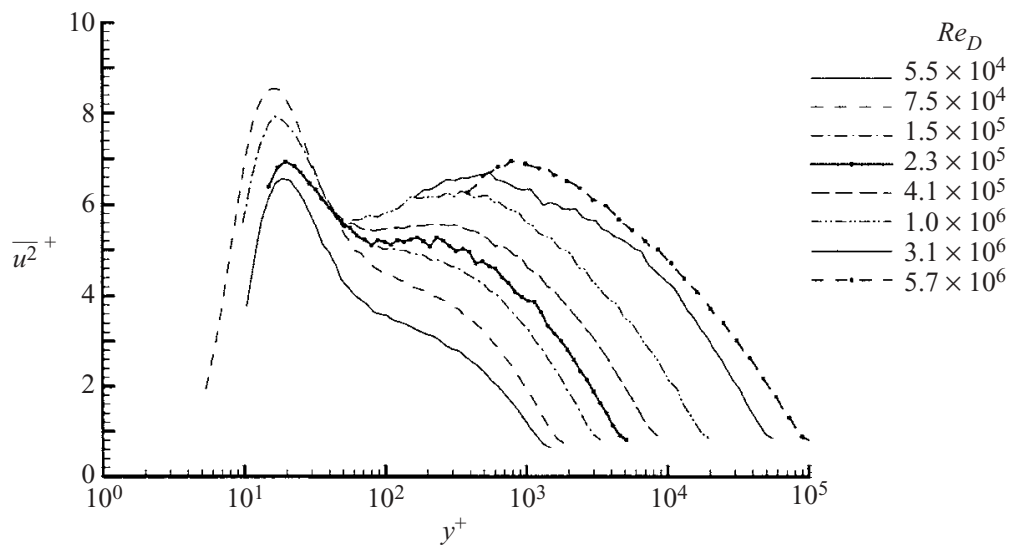

FIGURE 1. Second moment: wall scaling.

This should be regarded as a minor limitation of the thermal anemometry technique only (klewicki \& Falco 1990 find a similar result) rather than an indication of any anomaly in the current data sets. Near the wall, convergence improves as the Reynolds number increases, probably because of spatial filtering. However, outside of the range $10 \leqslant y^{+} \leqslant 30$, convergence does not improve with increasing Reynolds number and this indicates that all the averaging times are sufficient (table 1). The reduced averaging times at higher Reynolds number are simply the result of the faster sample rate and the resulting larger data sets. Even these averaging times are significantly longer than those suggested by Klewicki \& Falco (1990) for measurements of up to fourth-order moments in a boundary layer $\left(T U_{e} / \delta=4000\right)$ and Blackwelder \& Eckelmann (1979) for fourth-order velocity statistics in pipe flow $\left(T U_{c l} / R=2870\right)$.

\section{Results}

4.1. Spectra and second moments
The second moment, $\overline{u^{2}}$, normalized by $u_{\tau}^{2}$ and plotted against $y^{+}$shows two maxima (figure 1): the first, prevalent at low Reynolds numbers, is well-documented (Laufer 1954; Sandborn 1955; Eggels et al. 1994; Durst et al. 1995). The data of den Toonder \& Nieuwstadt (1997) (obtained using LDA) suggest that $\overline{u^{2}}$ reaches a maximum of about 7.3 that is constant with Reynolds number up to about $2.5 \times 10^{4}$ (see also Mochizuki \& Nieuwstadt 1996; Durst et al. 1995). However, the present data show that this maximum is, in fact, Reynolds-number dependent, reaching 8.6 at $R e_{D}=7.5 \times 10^{4}$. At higher Reynolds numbers (at $R e_{D}=1.5 \times 10^{5}, l^{+} \approx 25$ ), the reduction in this peak with increasing Reynolds number is, of course, the result of poorer spatial resolution. Ligrani \& Bradshaw (1987) note that measurements of $\overline{u^{2}}$ are significantly attenuated for $l^{+}>20-25$. The peaks at $R e_{D}=5.5 \times 10^{4}$ and $7.5 \times 10^{4}$ have values of 6.6 and 8.5 , respectively. For comparison, the nearwall peak at $R e_{D}=5.0 \times 10^{4}$ measured by Laufer (1954) has a value of 6.9 , and that measured by Perry \& Abell (1975) at $R e_{D}=7.8 \times 10^{4}$ has a value of 9.5 . The Reynolds-number dependence of the near-wall peak is, in some sense, consistent with the concept of inactive motion, namely that it increases with Reynolds number our first principal conclusion. Mochizuki \& Nieuwstadt (1996) suggest that the position of this peak is also independent of Reynolds number at $y^{+} \approx 15$. The present data do not contradict this, although owing to the effects of probe resolution, no 


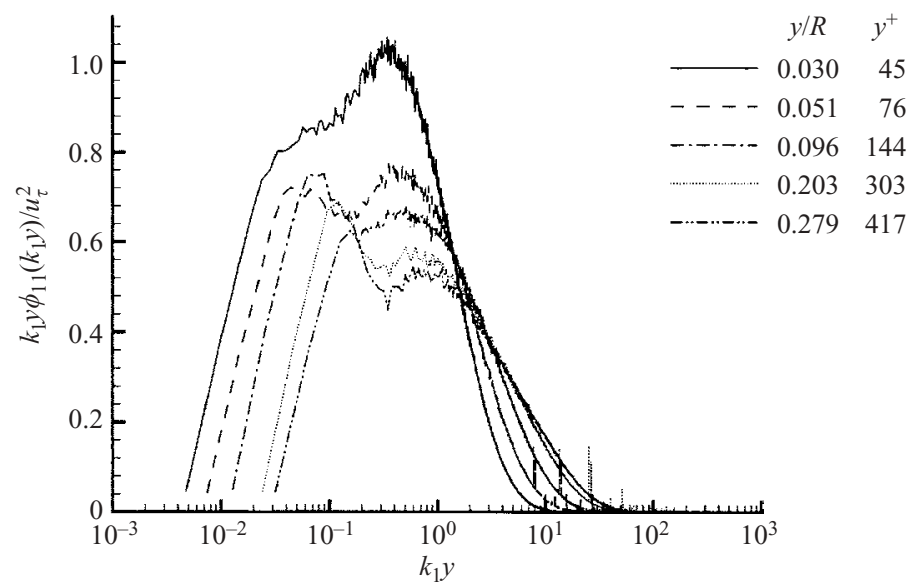

FiguRE 2. Inner scaling, $R e_{D}=5.50 \times 10^{4}, R^{+}=1.50 \times 10^{3}$.

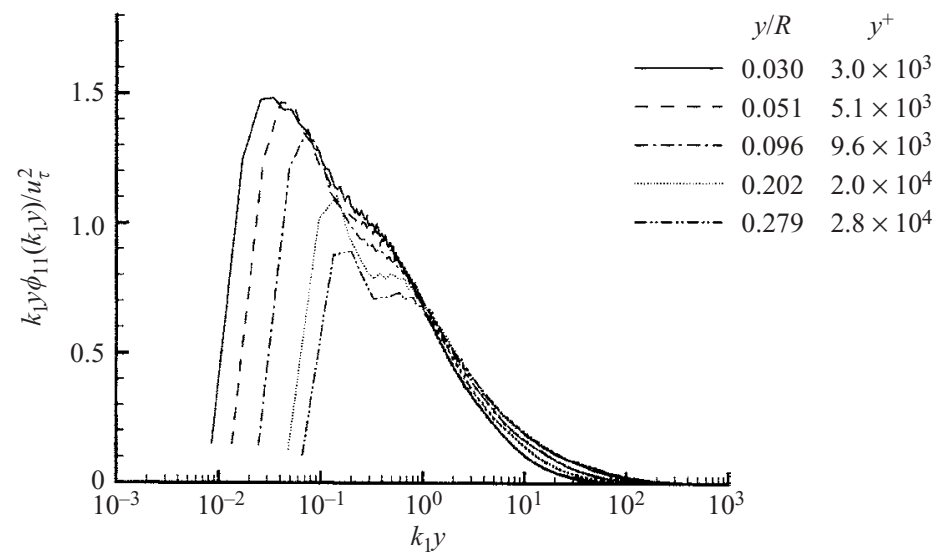

FIGURE 3. Inner scaling, $R e_{D}=5.7 \times 10^{6}, R^{+}=10^{5}$.

firm conclusions may be drawn. In the context of pipe flow, the second maximum at $y^{+} \approx 500$ and appearing only for $R e_{D}>2 \times 10^{5}$ is altogether a new phenomenon although its appearance in boundary layer data is well-documented: comparison of boundary-layer laboratory data (Fernholz \& Finley 1996) with those from the atmospheric surface layer (Marusic \& Kunkel 2003) suggest that the magnitude of the second maximum increases indefinitely with Reynolds number. Its position, $y_{p}^{+}$, also increases with Reynolds number. We examine the behaviour of both peaks in more detail in $\S 5$.

Using inner scaling, figure 2 shows $\phi_{11}\left(k_{1} y\right)$ in the form given by (2.7) for $R e_{D}=5.5 \times 10^{4}$ over the range in $y$ for which collapse might be expected. Note that this corresponds to a range of $y^{+}$over which the first moment displays a power law. Figure 3 shows equivalent data for $R e_{D}=5.7 \times 10^{6}$ plotted in the same form. In this case, the range of $y^{+}$corresponds to that of the log law except for the two points furthest from the wall. In figure 2, it is evident that the Reynolds number is simply too low for collapse to be possible. Note that $R^{+}=1500$ only and that the direct effects of viscosity permeate the whole layer. At the highest Reynolds number (figure 3 ), there is some collapse in the region $k_{1} y \approx 1.0$. However, the collapse is not 


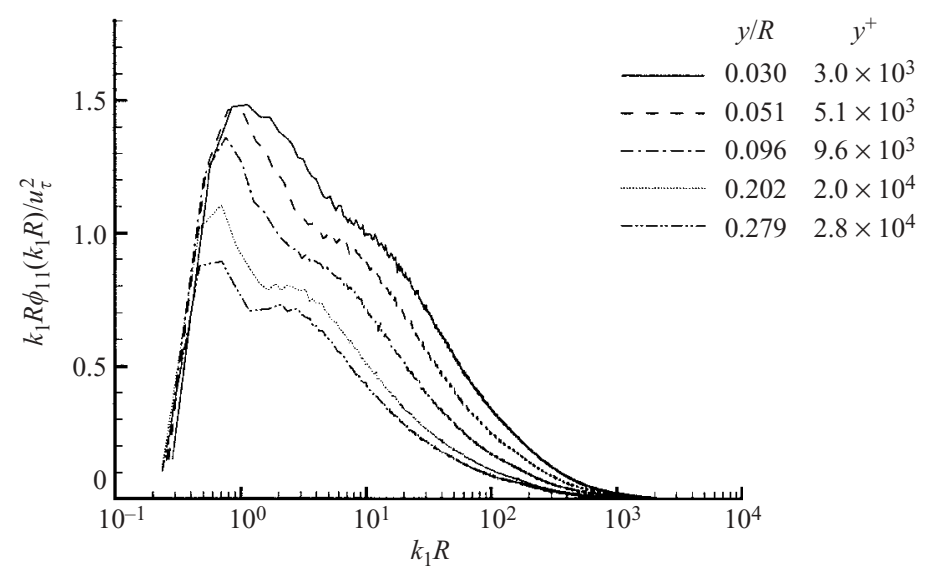

FIGURE 4. Outer scaling, $R e_{D}=5.7 \times 10^{6}, R^{+}=10^{5}$.

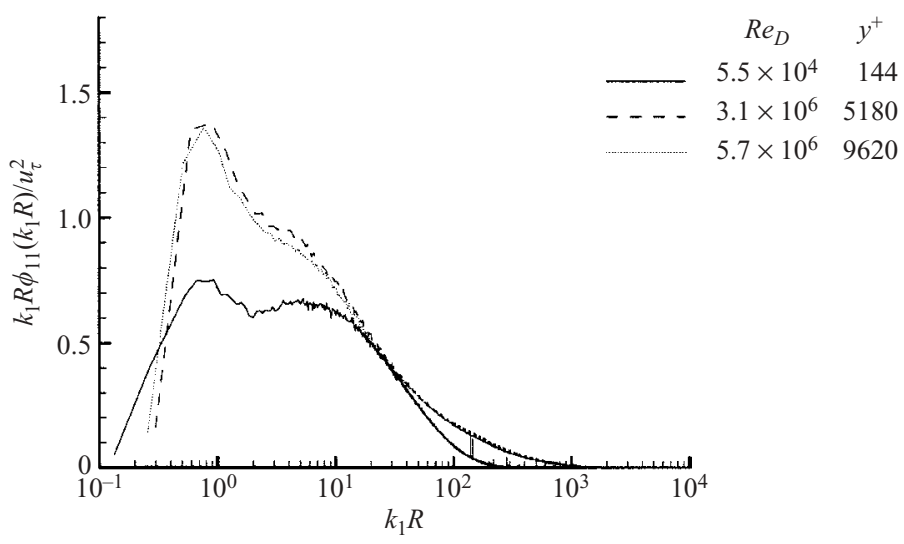

FIGURE 5. Outer scaling, $y / R=0.1$.

along a horizontal line (corresponding to self-similar scaling), suggesting incomplete similarity only.

Figure 4 shows the same data as in figure 3, but plotted using outer scaling. For $k_{1} R \sim 1$, there is no significant collapse, although the peaks for $0.033 \leqslant y / R \leqslant 0.107$ lie closer together than the others. Inspection of figure 3 in the region of $k_{1} y \sim 0.1$ shows that the same data $(0.033 \leqslant y / R \leqslant 0.107)$ clearly do not collapse using inner scaling. Instead, spectra for $y / R=0.033,0.063$ and 0.107 show discrete peaks, appearing in a wavenumber sequence determined by $y^{-1}$, equivalent to the collapse in figure 4 occurring at a point, $k_{1} R \approx 0.75$. Since collapse occurs neither with outer variables nor with inner variables, not even incomplete similarity is possible. At best, incomplete similarity is apparent only for $k_{1} R \leqslant 1$ using outer scaling, and for $k_{1} y \geqslant 0.06$ using inner scaling.

As suggested in $\S 2$, an alternative scrutiny of the data may be achieved by using outer scaling at different Reynolds numbers while fixing $y / R=0.1$. In figure 5, there is some degree of collapse for $k_{1} R \approx 0.8$ at the two higher Reynolds numbers. However, the equivalent data scaled with inner variables (figure 3 ) do not collapse so that again, at best, only incomplete similarity is possible. Note also that complete similarity would 


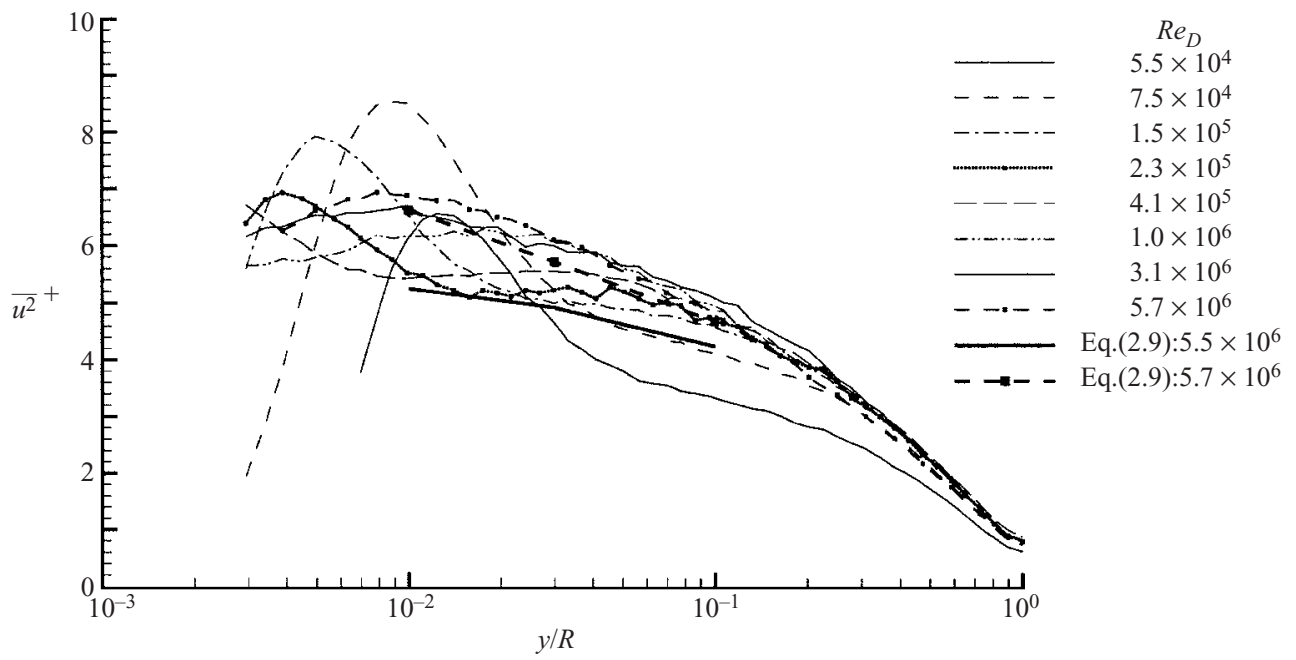

FIGURE 6. Second moment: outer scaling.

imply that $A_{1}$ takes a universal value, but it is clear from all the spectra that this is not the case.

Interestingly, as has been shown by Kim \& Adrian (1999) and Jiménez (1998), the spectra show the presence of very long structures near the wall that give rise to a bimodal shape at low Reynolds numbers, figure 2 . Their wavelength increases as $y$ increases, reaching a peak of about $10 R$ at $y / R \approx 0.1$ before decreasing at larger $y$. Figure 5 suggests that the length of these structures at a fixed $y$ does not change with Reynolds number (see Jiménez, Flores \& García-Villalba 2002).

Table 1 indicates that, at the highest Reynolds number, $R e_{D}=5.7 \times 10^{6}, l^{+}=385$. It is therefore important to assess the effect of hot-wire spatial averaging on the spectra of figures 3 and 4 , for which the critical parameter is $\left.k_{1} l\right|_{\max }=2 \pi$. For the spectra in figure 3 , this suggests full spectral resolution up to $\left.k_{1} y\right|_{\max }=2 \pi y / l=49,83,156,330$ and 454 for, respectively, positions $y / R=0.030-0.279$. Our inference is therefore that these spectral estimates (and, in particular, the deductions from them concerning self-similarity) are free of the effects of limits to spatial resolution. The effect of spatial resolution on ${\overline{u^{2}}}^{+}$is dealt with in $\S 5$.

Figure 6 shows the data of figure 1 re-plotted against $y / R$. There is a striking collapse in the outer region, $y / R>0.4$, except at the lowest Reynolds number. There is no collapse in the overlap region, and, as figure 1 shows, no collapse anywhere using wall variables, except presumably very close to the wall. Note that the near-wall peak now depends on $y / R$ : this provides a second principal conclusion, namely that inactive motion depends on distance from the wall. This is a result of general validity made trivial here because the near-wall peak in figure 1 occurs at approximately a constant value of $y^{+}$and $R$ is a constant. Figure 6 also shows a comparison with (2.9): at $R e_{D}=5.7 \times 10^{6}$ when the viscous deviation term is small, the agreement of the slope (determined by $A_{1}$ ) is quite good, but the offset (determined by the additive constant $B_{1}$ ) is too small. The changes with Reynolds number even at $y / R=0.1$ derive from the viscous deviation term, which qualitatively predicts the diminishing influence of viscosity outside the sublayer as the Reynolds number increases. At $R e_{D}=5.5 \times 10^{4}$ however, the behaviour of (2.9) at small $y / R$ is incorrect owing to the large inactive contribution. This is one of the two reasons for the change 
in gradient of the data (and therefore $A_{1}$ ) with Reynolds number. Moreover, for $0.02<y / R<0.1$, this change in gradient is not monotonic, first decreasing before increasing. This behaviour is therefore indicative of two effects: the increase in the inactive contribution with increasing Reynolds number (which is partly obscured by the poorer resolution as the Reynolds number increases), and the reduction in direct viscous effects emanating from the sublayer as the Reynolds number increases. While the latter is estimated quite well (but only for $R e_{D}>10^{6}$ ), no account of the former is taken in (2.9), which appears therefore to require an additional term quantifying the inactive contribution, the form of which is strongly dependent on the choice of outer velocity scale.

If a spectral self-similar range exists, such that $\phi_{11}\left(k_{1}\right) \propto k_{1}^{-1}$ ('complete' similarity), the constant of proportionality, $A_{1}$, in (2.9) is universal. However, the evidence of figure 6 is that the slope of the data in the vicinity of $y / R \approx 0.1$ (where the viscous deviation is negligible and where a $k_{1}^{-1}$ range is most likely) is still increasing at the highest Reynolds number. One should also bear in mind that $\overline{u^{2}}$, as the integral of $\phi_{11}$, is less sensitive to Reynolds-number scalings than the integrand itself. It is possible that at even higher Reynolds numbers, the slope of $\overline{u^{2}}$ may asymptote to a constant value indicative of complete similarity in $\phi_{11}$. With the omission of the viscous deviation term, (2.9) may be re-written as

$$
\overline{u^{2}}=B_{1}-A_{1} \ln \left[y^{+}\right]+A_{1} \ln \left[R^{+}\right] .
$$

This shows that the outer peak in figure 1 will increase indefinitely with Reynolds number, regardless of considerations of the universality of $A_{1}$. One might suppose that, at some stage, the outer peak might become larger than the inner peak in the sublayer. However, this is unlikely and it is more likely that the inactive motion near the wall will continue to increase with Reynolds number as long as its source in the outer region does. Using data from the atmospheric surface layer for which $R e_{\theta} \approx 5 \times 10^{6}$, Metzger \& Klewicki (2001) show that the near-wall peak does increase without any apparent indication of an asymptote.

Similarly, (2.8) may be rewritten as

$$
{\overline{u^{2}}}^{+}=B+A \ln \left[\frac{y}{R}\right]+A \ln \left[R^{+}\right]
$$

in which the offset, $a^{+}$, being much smaller than $y^{+}$is neglected. For clarity, the Reynolds-number dependence of $A$ and $B$ is not made explicit and we take their values to be approximately the same as those of Perry et al. (1986) since the functional forms of (4.2) and (2.9) are very similar. Thus $-A \approx A_{1}=0.9$ to ensure that the slope of $\bar{u}^{2}+$ is negative. It is hardly surprising therefore to find that (4.2) fails to represent $u^{2}$ well: it leads to negative values of $u^{2}$ when the $\ln \left[R^{+}\right]$term is large. The incorrect functional form of (4.2) is due to the supposed simple scaling of the normal stress with mean velocity.

\subsection{Probability density functions and higher moments}

We now examine the nature of the probability distribution functions (p.d.f.s) and their higher moments up to sixth order. Their departure from Gaussian and its ramifications are dealt with in $\S 5$.

Figure 7 shows selected p.d.f.s at $R e_{D}=4.1 \times 10^{5}$ for $y^{+} \geqslant 60$. This Reynolds number is just high enough for there to be a log region according to the criterion of Zagarola \& Smits (1998). The points chosen extend from $y^{+}=60$ where the data can 


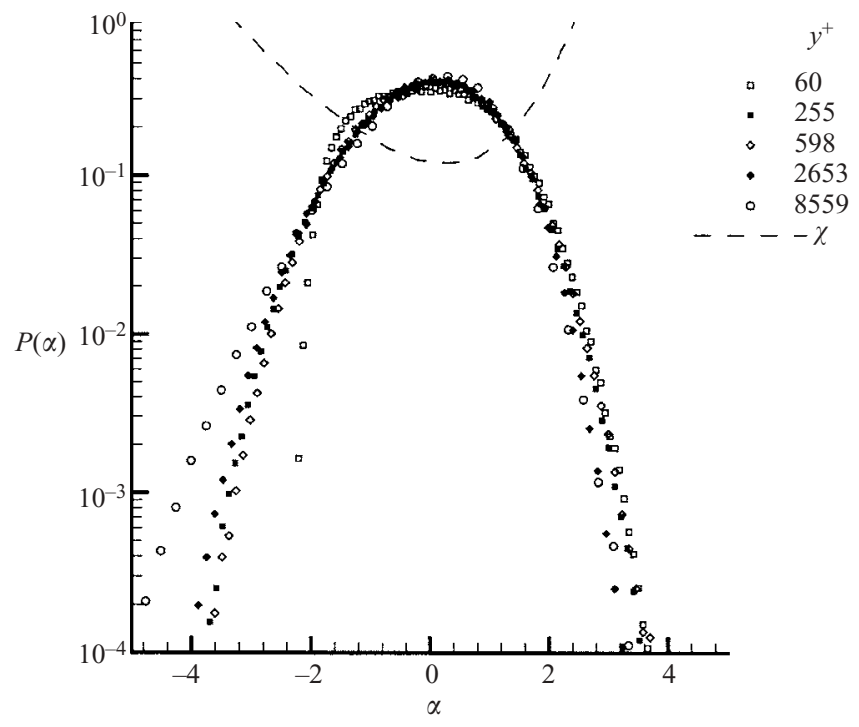

FIgURE 7. P.d.f.s of $u$ for $R e_{D}=4.1 \times 10^{5} . \chi$ for data at the centreline.

be expected to be substantially free of resolution effects. The p.d.f. is defined by

$$
P\left(u_{i}\right)=\frac{N_{i}}{N \Delta u}
$$

where $N$ is the total number of occurrences, $N_{i}$ is the number of occurrences for any velocity, $u_{i}$, and $\Delta u$ is the bin size. Here, normalization is performed with the first moment subtracted so that $\alpha=u / \sigma$ (where $\sigma$ is the standard deviation) and therefore

$$
\int_{-\infty}^{\infty} P(\alpha) \mathrm{d} \alpha=1 .
$$

Also shown are the estimates of r.m.s. relative error, $\chi$, at $y^{+}=R^{+}=8559$ as defined by Lumley (1970):

$$
\chi=\sqrt{\frac{2 T}{N_{i} \delta t}},
$$

where the integral timescale is estimated as $T=R / u_{\tau}$ and $\delta t$ is the sampling interval. At any position, $\Delta u$ is set by dividing the difference between the signal maximum and minimum into one hundred equal intervals. Thus $\chi$ is bound to be large at the extremes of the p.d.f., especially when the higher moments are numerically large, as occurs both near the wall and the pipe centreline. Figure 8 shows p.d.f.s for the range $5.5 \times 10^{4} \leqslant R e_{D} \leqslant 5.7 \times 10^{6}$ at $y^{+} \approx 600$, which is at the lower limit of the log-law region except for those data at $R e_{D}<4.1 \times 10^{5}$.

Using DNS data equivalent to $R^{+} \leqslant 395$, Dinavahi, Breuer \& Sirovich (1995) suggest that p.d.f.s of velocity fluctuations in turbulent channel flow exhibit universality in that, outside the viscous sublayer, they are independent of distance from the wall. In contrast, figure 7 shows that these p.d.f.s do not collapse: for example, they become progressively more negatively skewed with increasing distance from the wall. Moreover, the maximum value of $P$ changes from 0.38 at $y^{+}=60$ to 0.43 at the centreline. Dinavahi et al. (1995) also suggest that p.d.f.s obtained by averaging 


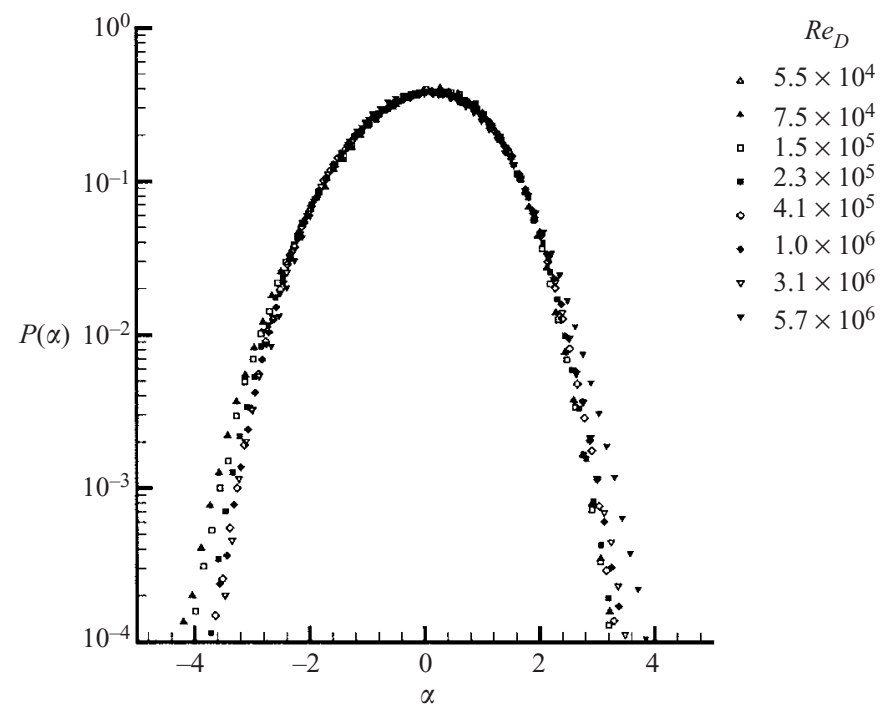

FiguRE 8. P.d.f.s of $u$ at $y^{+} \approx 600$.

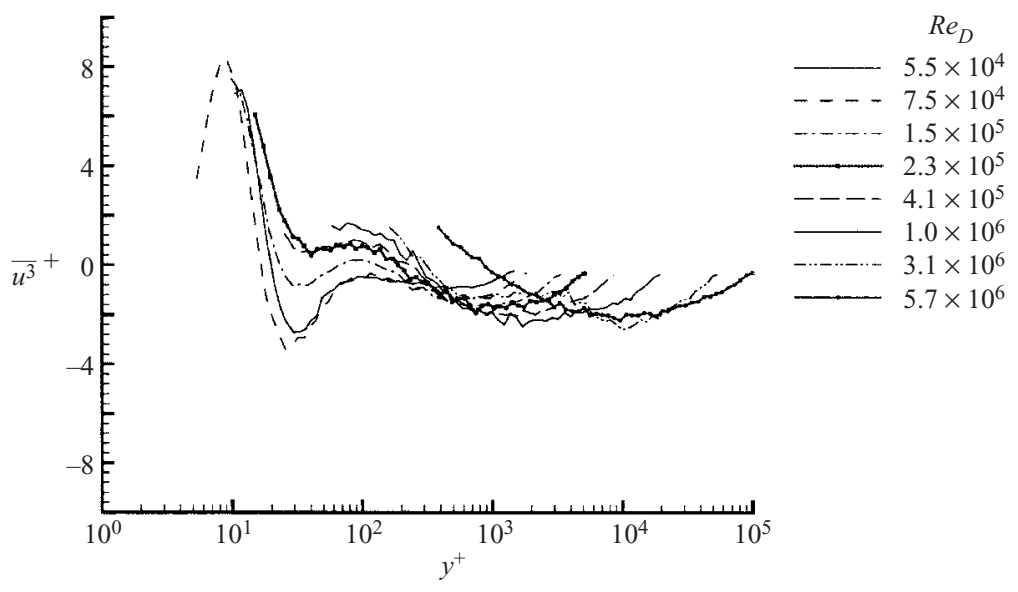

FIGURE 9. Third moment: wall scaling.

those at several distances from the wall in the range $63 \leqslant y^{+} \leqslant 318$, are 'relatively independent' of Reynolds number. It is clear that the p.d.f.s are neither constant with $y^{+}$at a given Reynolds number (figure 7) nor constant at a given $y^{+}$for a range of Reynolds numbers (figure 8), although in the case of the latter, the collapse is better. Generally, the differences become larger as the moment order increases.

Therefore a more precise assessment of the velocity field comes from examining the moments of the p.d.f.s. Figures 9-12 show, in sequence, moments from the third to the sixth scaled using wall variables. While the even moments are similar in shape to each other (figures 1,10 and 12), the odd moments are also similar to one another (figures 9 and 11). Thus the behaviour of the peaks in ${\overline{u^{2}}}^{+}$is very similar to that for the fourth and sixth moments. There is no scaling with wall variables anywhere. 


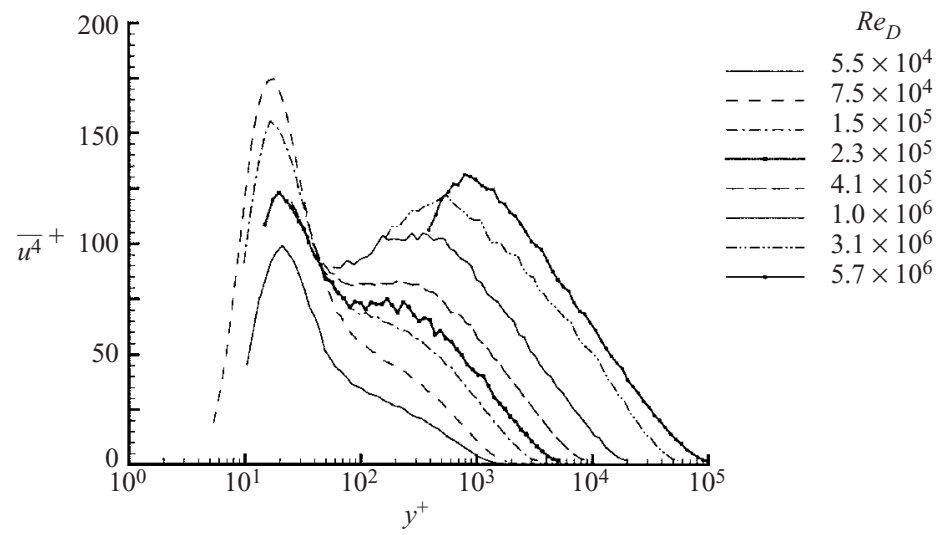

FIGURE 10. Fourth moment: wall scaling.

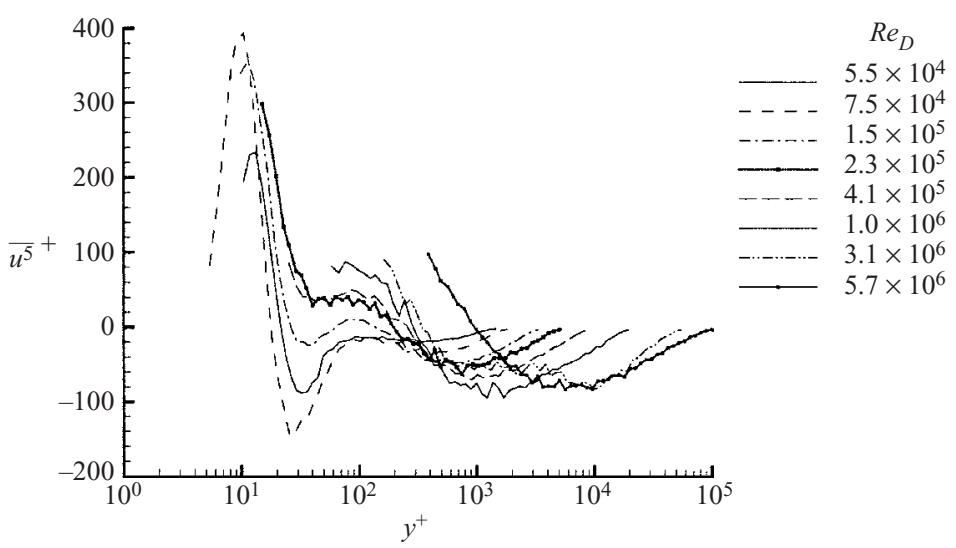

FIGURE 11. Fifth moment: wall scaling.

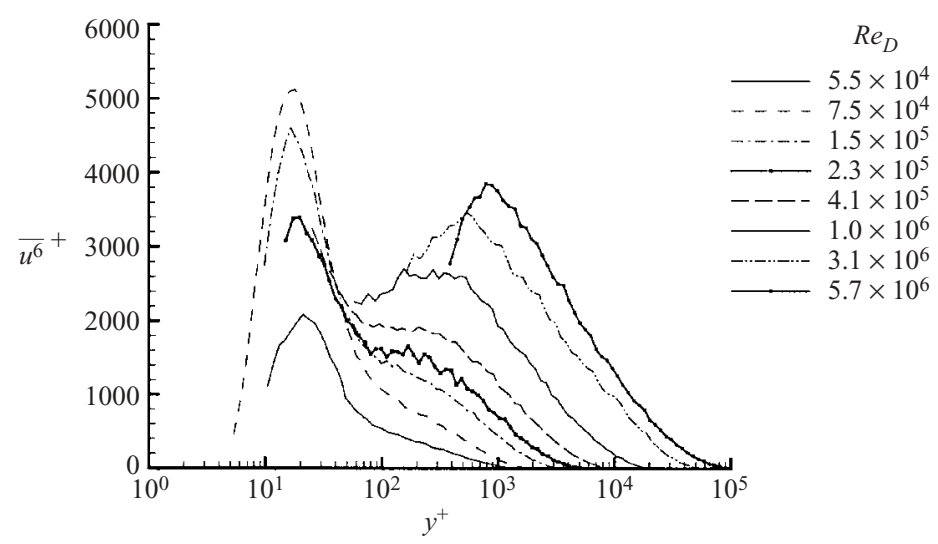

FIGURE 12. Sixth moment: wall scaling.

In order to compare statistics with those for a Gaussian p.d.f., the same moments are scaled using the appropriate power of the second moment. Figures 13-16 show the skewness, $S_{u}$, flatness, $F_{u}$, superskewness, $S S_{u}$ and superflatness, $S F_{u}$, respectively, plotted against $y^{+}$. As den Toonder \& Nieuwstadt (1997) suggest, these statistics 


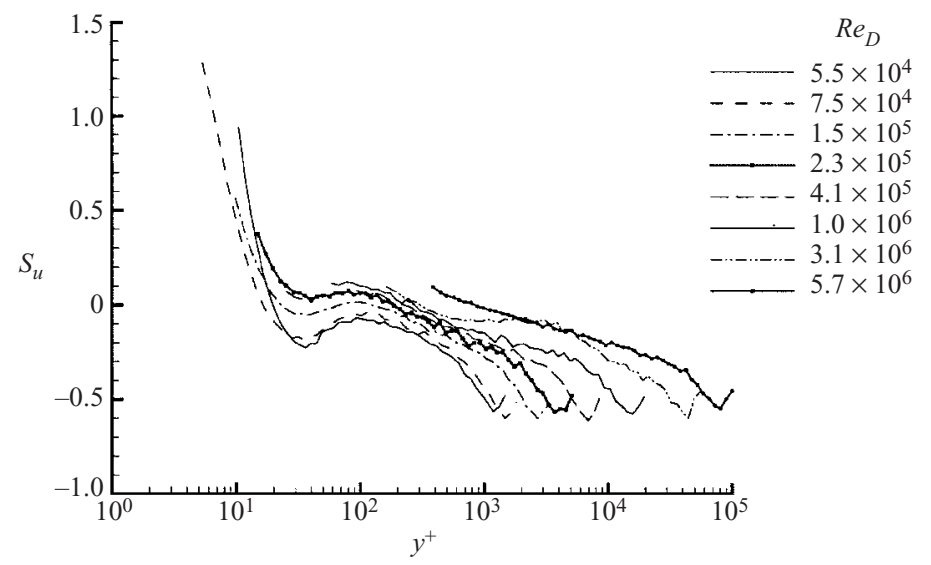

FIGURE 13. Skewness: wall scaling.

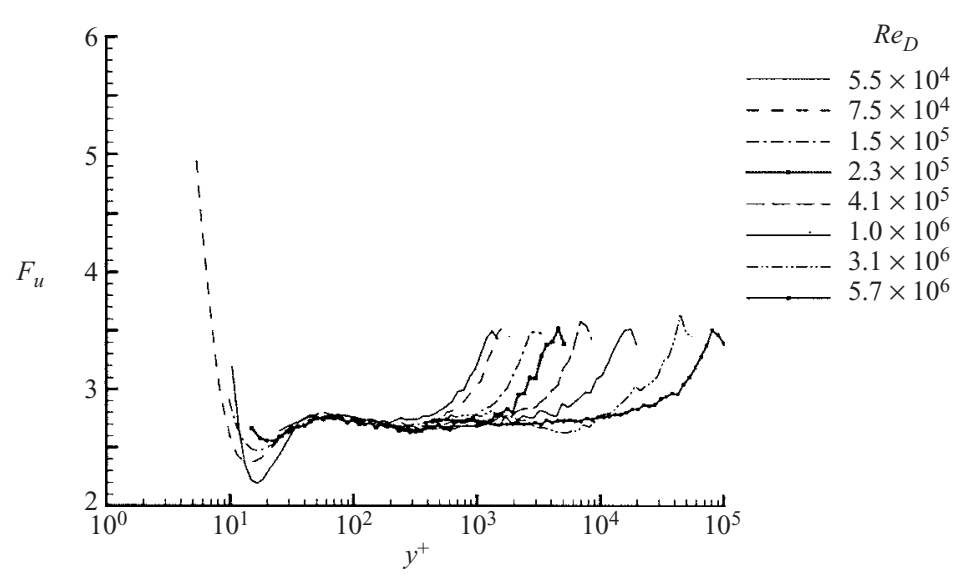

Figure 14. Flatness: wall scaling.

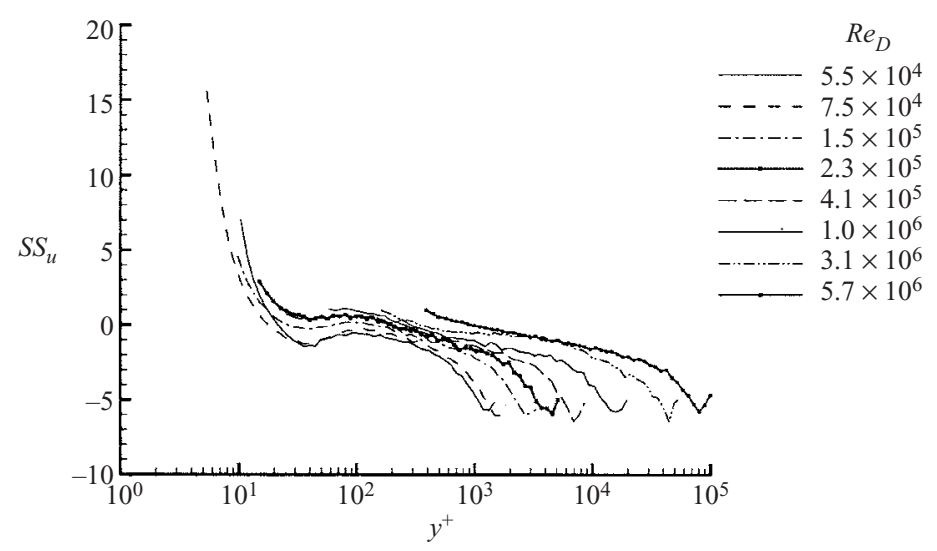

FIGURE 15. Superskewness: wall scaling.

are Reynolds-number dependent even though the changes are modest. However, $F_{u}$ and $S F_{u}$ show very good collapse for $30 \leqslant y^{+} \leqslant 300$ and, at the higher Reynolds numbers, the position of the outer peak of ${\overline{u^{2}}}^{+}, y_{p}^{+}$, lies within the region of collapse. 


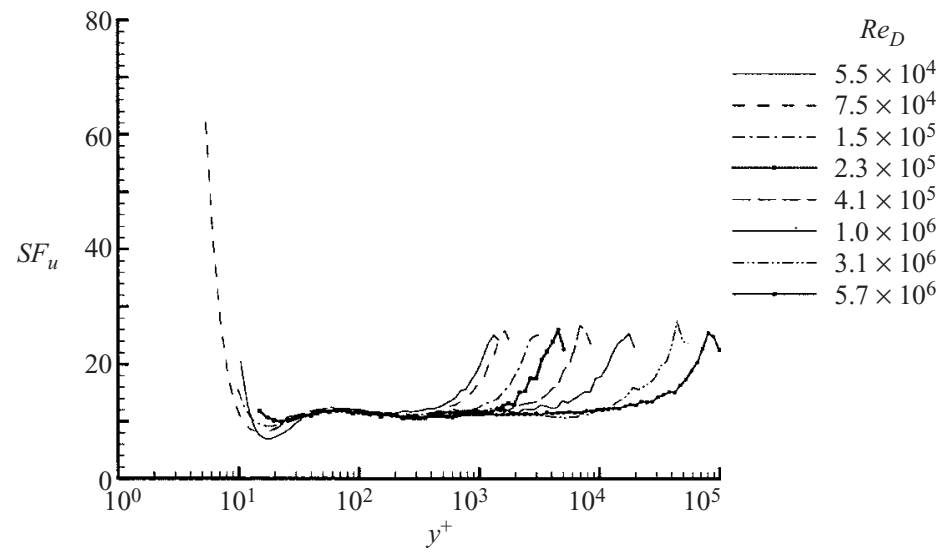

FIGURE 16. Superflatness: wall scaling.

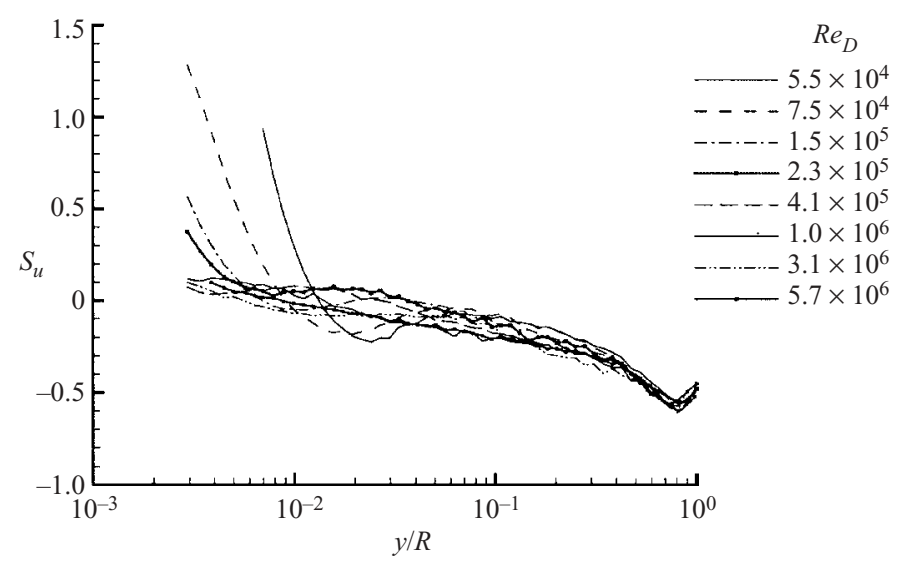

FIGURE 17. Skewness: outer scaling.

Note that, as elsewhere, in this region none of the statistics have Gaussian values $\left(S_{u}=S S_{u}=0, F_{u}=3\right.$ and $\left.S F_{u}=15\right)$. Near the wall, $S_{u}$ and $F_{u}$ are both large and positive, while near the centreline, $S_{u}$ is large and negative and $F_{u}$ is again large and positive. Physically, eruptions from the near-wall region arrive at the centreline with low streamwise momentum, and conversely, wallward-moving eddies arrive at the wall with high streamwise momentum. Any changes with Reynolds number of the former effect cannot be deduced owing to the effects of probe resolution. However, at the lowest Reynolds number, $S_{u}$ is still increasing at smaller values of $y^{+}$than that at which $\overline{u^{2}}$ reaches its sublayer maximum, at which point $\left(y^{+} \approx 15\right), S_{u}$ is close to zero $( \pm 0.2)$ as is $S S_{u}( \pm 1.0)$. Meanwhile, $F_{u}(\approx 2.4)$ and $S F_{u}(\approx 9.0)$ both exhibit minima here, as suggested by den Toonder \& Nieuwstadt (1997) and Durst et al. (1995). Note however, that these values are also non-Gaussian.

For brevity, we show only $S_{u}$ and $F_{u}$ plotted against $y / R$ in figures 17 and 18 . As with inner scaling, the degree of collapse is better for even moments than odd ones, but generally, it improves with increasing moment order and increasing $y / R$. At $y / R \approx 0.8, S_{u}$ show minima and $F_{u}$ show maxima. As will be seen later, this is not an indication of asymmetry, merely that the point of maximum departure from Gaussian occurs at $0.2 R$ either side of the centreline. This is probably due to the 


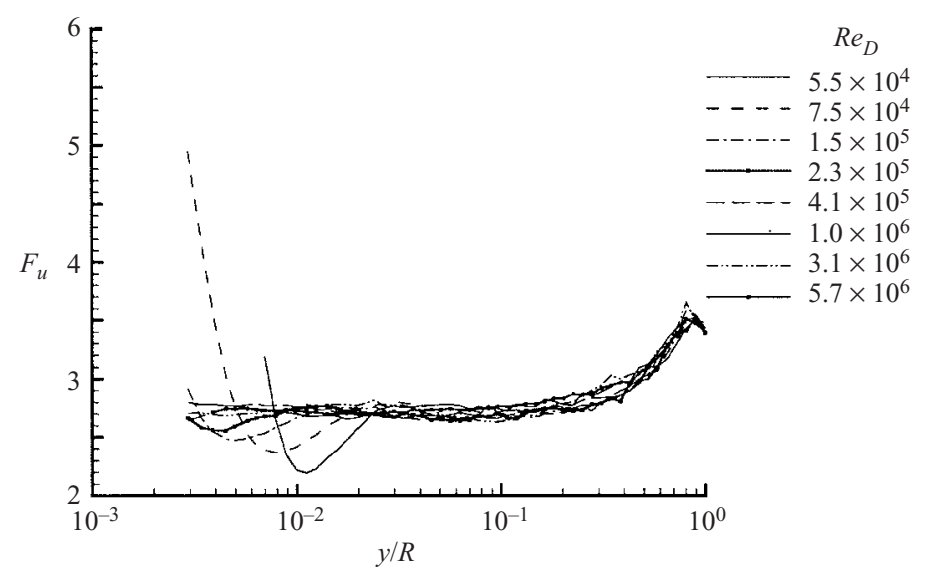

FIGURE 18. Flatness: outer scaling.

influence of the 'geometry effect' (Wei \& Willmarth 1989), or its equivalent in pipe flow, in which eddies from all azimuthal locations converge on the centreline. The changes in moment behaviour for $0.2 R$ either side of the pipe axis are consistent with the increasing influence of fast-moving eddies oscillating about the centreline with a frequency of occurrence that is slightly less than that of near-wall eruptions reaching the centreline. Interestingly, this effect appears not to diminish as the Reynolds number increases, as evidenced by the persistence of outer scaling down to about $0.4 R$. Without reference to the wall-normal velocity component, it is not possible to say over what range of radial positions the geometry effect is prevalent.

Tsuji \& Nakamura (1999) have proposed the use of the Kullback Leibler Divergence to measure the departure of p.d.f.s of the streamwise velocity from Gaussian in a turbulent boundary layer. They suggest that the extent of the log law is defined as that over which the p.d.f. is 'similar' when normalized by the second moment. Given the difficulties mentioned in $\S 2$ of using the first moment to make inferences about the behaviour of the higher ones (or vice versa), here we test this assertion for the present pipe flow in which the extent of the log-law region has been closely delineated (Zagarola \& Smits 1998; McKeon et al. 2004). In the range $600 \leqslant y^{+} \leqslant 0.12 R^{+}$, from the results of figures 17 and 18 , it is clear that, although $S_{u}$ and $F_{u}$ are relatively constant, their variation is such that this definition is unlikely to be as rigorous as that provided by any form of overlap analysis (Zagarola \& Smits 1998; Wosnik et al. 2000; McKeon et al. 2004). Indeed, all of the results here are consistent with the notion that the turbulence, as exemplified by the second and higher moments of the streamwise velocity, does not show any self-similarity. The departure of these statistics from Gaussian is dealt with more fully in the next section.

\section{Discussion}

\subsection{Alternatives to $u_{\tau}$ as a velocity scale}

Zagarola \& Smits (1998) have used the data of den Toonder \& Nieuwstadt (1997) to show that $U_{c l}-\bar{U}$ collapses the second moment better than $u_{\tau}$ for $0.2<y / R<1.0$, for the range $4.9 \times 10^{3} \leqslant R e_{D} \leqslant 2.5 \times 10^{4}$. Note that $U_{c l}-\bar{U}$ is frame-invariant. Here we test this velocity scale using data over a much wider range of Reynolds numbers. Given the lack of success in the use of $u_{\tau}$ to collapse moments in the inner region, mixed scaling (DeGraaff \& Eaton 2000; Metzger et al. 2001) as an alternative is 


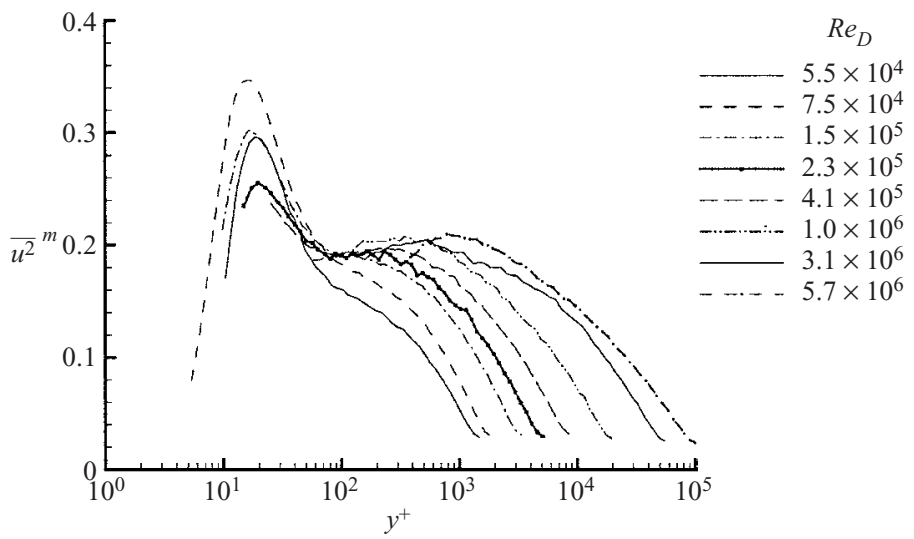

FiguRE 19. Second moment: mixed scaling, $y^{+}$abscissa.

also assessed. This is especially important because evidence of an inner velocity scale that is different from the outer one immediately prevents self-similarity for the velocity spectrum in terms of a $k^{-1}$ region. It also raises questions concerning the physical mechanisms that might give rise to velocity scales other than $u_{\tau}$ : for instance, $U_{c l}-\bar{U}$ as a velocity scale would suggest freely moving, convected large structures (see Morrison et al. 1992). We do not consider further the analysis of Tsuji \& Nakamura (1999) who use an expression for $\overline{u^{2}}$ in the log-law region that only applies if $\overline{u^{2}}+$ decreases monotonically.

\subsubsection{Inner velocity scale}

As a justification for mixed scaling, DeGraaff \& Eaton (2000) suggest, following Rotta (1962), that the total rate of energy dissipation in a boundary layer depends on $u_{\tau}^{2} U$. Rotta (1962) suggests that a good approximation to the total energy balance close to the wall is given by

$$
u_{\tau}^{2} \frac{\mathrm{d} U}{\mathrm{~d} y}=-\overline{u v} \frac{\mathrm{d} U}{\mathrm{~d} y}+v\left(\frac{\mathrm{d} U}{\mathrm{~d} y}\right)^{2},
$$

where the left-hand side may be taken to be the transport of energy from the outer region to the wall region. However, the results of $\S 4$ suggest that that the left-hand side of (5.1) is more likely to scale as $u_{\tau}^{3} / y$ and not $u_{\tau}^{2} U_{c l} / y$. Note that the local-equilibrium hypothesis (a result of applying wall scaling to the turbulence kinetic energy equation) suggests $\epsilon \propto u_{\tau}^{3} / y$. Such a scaling is consistent with the notion that both active and (to a first approximation) inactive components arise through the presence of attached wall eddies. As we have seen in $\S 2$, a particular problem with the choice of a velocity scale involving any mean velocity is that it is frame-dependent. Moreover, there is no physical reason why the geometric mean of two velocity scales might be appropriate. Thus the use of $\left(u_{\tau} U_{c l}\right)^{1 / 2}$ would appear to have little physical basis. Leaving aside the issue of a corresponding mixed lengthscale, ${\overline{u^{2}}}^{m}\left({\overline{u^{2}}}\right.$ normalized by $\left.u_{\tau} U_{c l}\right)$ is plotted against $y^{+}$in figure 19 and against $y / R$ in figure 20. In the case of the former, the collapse is no better than that of wall scaling in figure 1, a result that is apparently at odds with the conclusion of Metzger et al. (2001) using data from the atmospheric surface layer. However allowing for the poorer resolution in the present data, the changes in ordinate value at $y^{+} \approx 15(0.3-0.4)$ are similar in both cases. This suggests that the choice of $U_{c l}$ masks a more subtle effect. In the case of figure 20, the collapse 


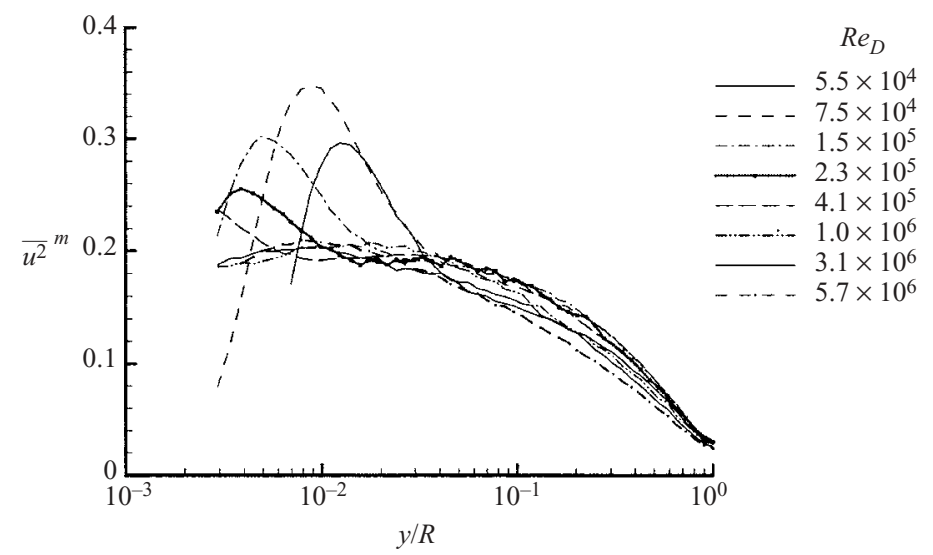

FiguRE 20. Second moment: mixed scaling, $y / R$ abscissa.

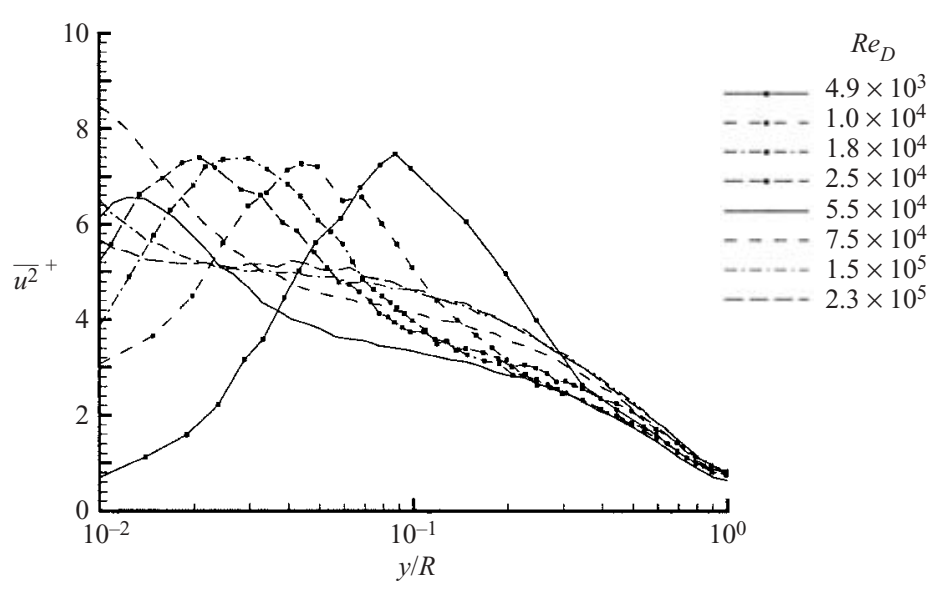

FIGURE 21. Second moment: outer scaling, $u_{0}=u_{\tau}$. Legend shows data in sequence of increasing Reynolds number. Solid symbols indicate data from den Toonder \& Nieuwstadt (1997).

is demonstrably worse than the outer scaling of figure 6. Similarly, there is no collapse when using the mean velocity as a velocity scale (not shown) except at the centreline.

\subsubsection{The outer velocity scale}

Since $u_{\tau}$ and $U_{c l}-\bar{U}$ are related only by a constant of proportionality for $R e_{D}>2 \times 10^{5}$ (McKeon et al. 2004), the question of the better velocity scale relates to those data for $R e_{D}<2 \times 10^{5}$ only, that is, the data sets at the four lower Reynolds numbers. In order to extend the comparison of velocity scales over a wider range of Reynolds numbers, ${\overline{u^{n}}}^{+}$and ${\overline{u^{n}}}^{\Delta}={\overline{u^{n}}}^{n} /\left(U_{c l}-\bar{U}\right)^{n}$ are compared using the present data for $\operatorname{Re}_{D} \leqslant 2.3 \times 10^{5}$ together with those of den Toonder \& Nieuwstadt (1997), for which $4.9 \times 10^{3} \leqslant R e_{D} \leqslant 2.5 \times 10^{4}$.

Figures 21-23 show, respectively, second-, third- and fourth-order moments, scaled with outer scales taking $u_{o}=u_{\tau}$. There is a striking collapse of all moments in the outer region for $y / R>0.4$. There is no collapse in the overlap region, as there is no collapse using inner variables (figures 1 and $7-12$ ) - the overlap region relates only to the mean velocity and not to the turbulence statistics. Note that the inward progression 


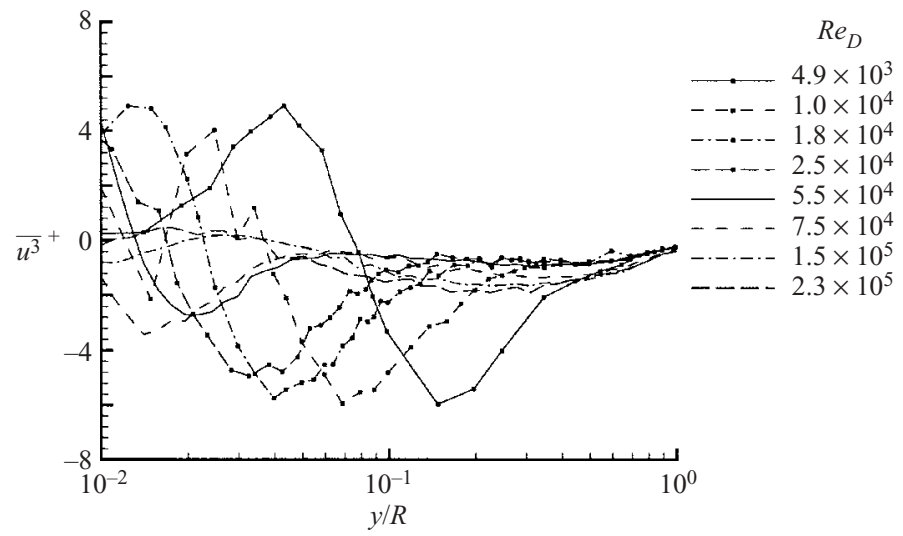

FIGURE 22. Third moment: outer scaling, $u_{0}=u_{\tau}$.

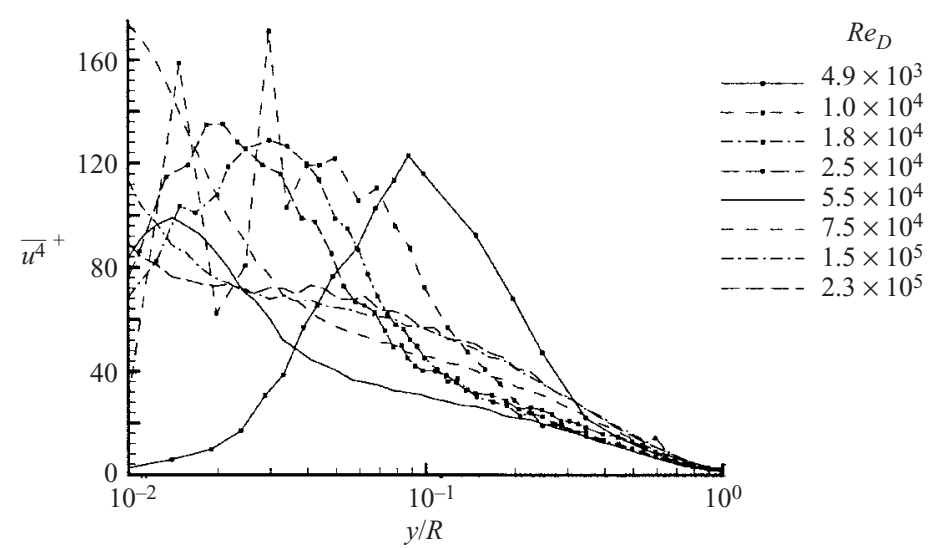

FIGURE 23. Fourth moment: outer scaling, $u_{0}=u_{\tau}$.

of the peak in both ${\overline{u^{2}}}^{+}$and ${\overline{u^{4}}}^{+}$is consistent with the Reynolds-number changes over both data sets.

The same data are re-plotted with $u_{0}=U_{c l}-\bar{U}$ in figures 24-26 where estimates of $U_{c l}-\bar{U}$ for the present data are estimated from a curve fit to the data of Zagarola \& Smits (1998). Use of the equivalent data from McKeon et al. (2004) gives a change in $u_{\tau}$ of about $0.3 \%$, which is very close to the expected error in $u_{\tau}$ (Zagarola \& Smits 1998). Comparison of figures 21 and 24, of figures 22 and 25, and of figures 23 and 26 is especially revealing: it supports the assertion by Zagarola \& Smits (1998) that $U_{c l}-\bar{U}$ is a better outer velocity scale than $u_{\tau}$ for the second moment for $y / R>0.35$, approximately. While the triple product appears to scale equally well with either velocity scale, the fourth-order moment collapses better with $U_{c l}-\bar{U}$. However, the improved scaling only appears to affect the data for $R e_{D} \leqslant 6 \times 10^{4}$ : that is, all of the data sets from den Toonder \& Nieuwstadt (1997) together with one of the present data sets at the lowest Reynolds number. In figures 24 and 26, the behaviour almost suggests two separate ranges of Reynolds number over which the data collapse for $y / R>0.35$ : one for $R e_{D}<6 \times 10^{4}$, the other for $R e_{D}>7 \times 10^{4}$. Note that this result is unlikely to affect the dimensional arguments for a $k_{1}^{-1}$ range in the spectra since the 


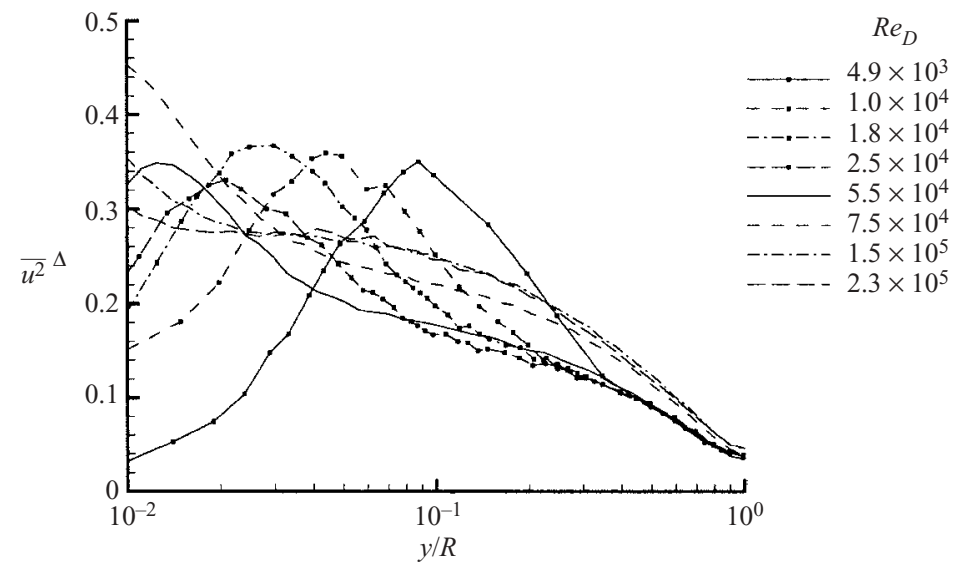

FIGURE 24. Second moment: outer scaling, $u_{0}=U_{c l}-\bar{U}$. Solid symbols indicate data from den Toonder \& Nieuwstadt (1997).

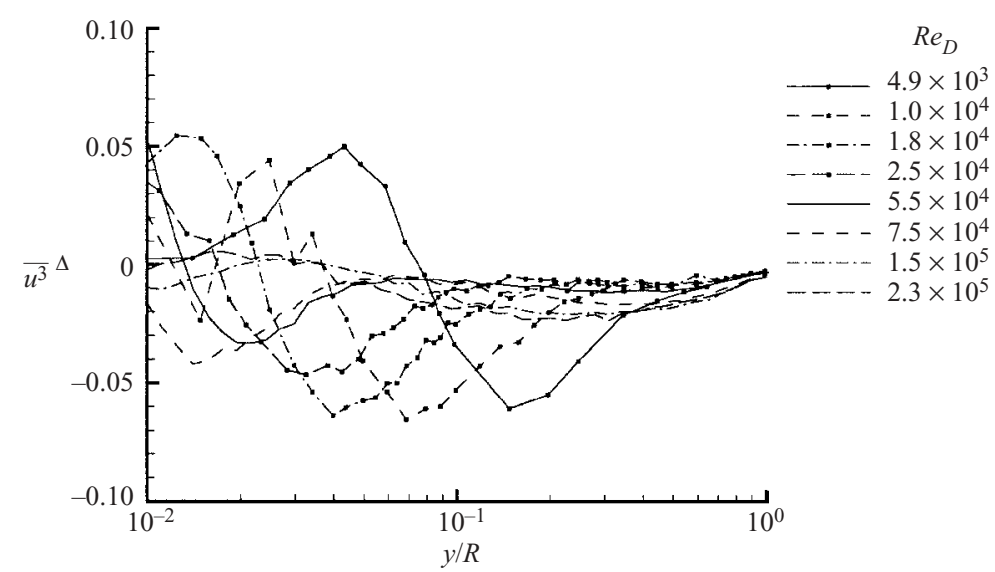

FIGURE 25. Third moment: outer scaling, $u_{0}=U_{c l}-\bar{U}$.

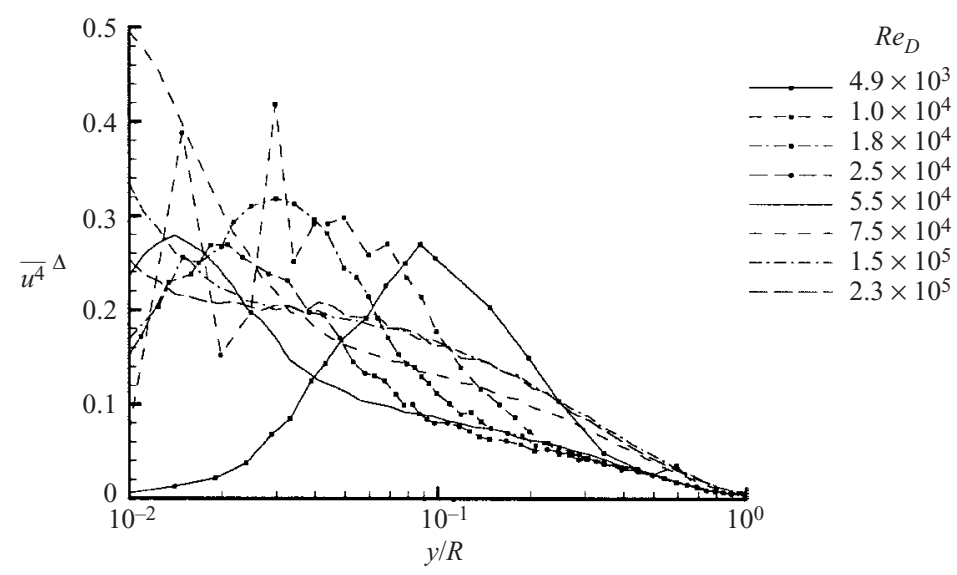

FIGURE 26. Fourth moment: outer scaling, $u_{0}=U_{c l}-\bar{U}$. 
ratio of $U_{c l}-\bar{U}$ to $u_{\tau}$ reaches a constant for $R e_{D}>2 \times 10^{5}$ and the choice of velocity scale does not matter as long as it is invariant with Reynolds number. It would also suggest that the outer region is more likely to be dominated by large, freely moving detached structures, or at least those that do not conform to Townsend's concept of an attached wall eddy. This suggestion may well be realistic: Perry \& Marusic (1995) and Marusic \& Perry (1995) show that prediction of the Reynolds stresses using their attached wall eddy still requires an additional component more like a detached structure.

\subsection{Self-similarity}

We have illustrated the importance of, first, the direct effects of viscosity outside the viscous sublayer at low Reynolds numbers and, second, the influence of inactive motion at high Reynolds numbers. Our most important result is perhaps that, while the concept of inactive motion serves as a very useful qualitative description of the low-wavenumber motion near the wall, it is somewhat of a misnomer. Westbury \& Morrison (1995) have previously noted that the distinction between the large-scale inactive motion and the associated shear-stress-bearing sweeps is not necessarily as clear cut as Townsend's ideas might suggest. The evidence of figure 1 is that inactive motion increases $\overline{u^{2}}$ just where the production of turbulence kinetic energy reaches a maximum. Thus the outer eddies are, in fact, active in terms of producing energy by the shear that they induce. This result underscores the importance of the 'top-down' influence at high Reynolds numbers identified by Hunt \& Morrison (2000). While the alternative 'bottom-up' effect, in which it is supposed that near-wall instabilities drive the momentum flux, may be an adequate description at very low Reynolds numbers, the foregoing suggests that these two effects may be reconciled by recognizing that the Reynolds number is a measure of the ratio of one to the other. This interpretation suggests that the near-wall motion is not autonomous as suggested by the studies of Jiménez \& Pinelli (1999) and Jiménez et al. (2002) using DNS data. More recently, Jiménez, del Alámo \& Flores (2004) have suggested that near-wall motion also consists of large structures that extend to the outer region. However, these structures carry little Reynolds stress (they are 'inactive' as Townsend suggested). This suggests that the technique used to mask the outer-region influence does not mimic the Reynolds-number effects reported here.

It has been suggested to us by some reviewers that a self-similar $k_{1}^{-1}$ range is more likely to exist closer to the wall than the range of $y^{+}$represented by the data of figure 3. This was examined in earlier data sets in Morrison et al. (2001) who show that for $y^{+}<1000$, the direct effects of viscosity prevent any form of self-similarity. The spectra of figures 3 and 4 therefore represent that range of $y$ over which self-similarity is most likely to be apparent.

It is interesting to note that, as suggested by figure 4 , the lower limit to the region in which complete similarity of the low-wavenumber motion is most likely to exist, $0.033 \leqslant y / R \leqslant 0.107$, is equivalent to $y^{+} \approx 5000$ at $R e_{D}=5.7 \times 10^{6}$, and that collapse of the spectra is significantly worse at lower Reynolds numbers. Thus it is very unlikely that complete similarity in the form of the $k_{1}^{-1}$ range will be possible below this Reynolds number. Note that an equivalent boundary-layer Reynolds number is $R e_{\theta} \approx 300000$ ! Figure 6 shows that the direct effects of viscosity on $\overline{u^{2}}$ are apparent in the outer region for $R e_{D}<10^{5}$. On balance, it would appear that while collapse of the velocity spectra may be possible with either inner or outer scaling (incomplete similarity), it is unlikely that simultaneous collapse with both in the same wavenumber range is possible (complete similarity), at least up to the maximum Reynolds number 
attained here. Thus spectra here do not exhibit a $k_{1}^{-1}$ range indicative of self-similar structure, which should therefore be considered as a special case only. The behaviour of $\overline{u^{2}}$ is consistent with the notions that $(a)$, inactive motion increases with Reynolds number and $(b)$ inactive motion increases as $y / R$ decreases (down to the sublayer). On the basis of $(a)$ and $(b)$ alone, complete similarity as outlined above seems to be unlikely because, while the active motion scales on $y$ and $u_{\tau}$ only (in the limit of infinite Reynolds number) as Townsend proposed, the inactive component always requires three scales, namely $y, R$, and a velocity scale, in compliance with $(a)$ and (b) above.

It is possible that a $k_{1}^{-1}$ range may appear at even higher Reynolds numbers typical of the atmospheric surface layer, and a complete analysis of atmospheric data is required (but see Marusic \& Kunkel 2004). Its often-published appearance (see, for instance, Kader \& Yaglom 1991; Katul \& Chiu 1998; Nikora 1999; Hunt \& Carlotti 2001; Högström et al. 2002) leads to the obvious question of why this might be so. Townsend $(1956$, p. 206) appears to have initially suggested the possibility of a $k_{1}^{-1}$ range without distinguishing complete and incomplete similarity. In fact, it would appear that Townsend (1976, p. 154) began to recognize that self-similarity was unlikely: 'It now appears that simple similarity of the motion is not possible with attached eddies and, in particular, that the stress-intensity ratio depends to some extent on position in the layer.' Despite this later change of view, the acceptance of a $k_{1}^{-1}$ range has continued with what now appears to be incomplete similarity only. As we have suggested, complete similarity requires a single velocity scale. In addition, it also implies that contributing structures are space-filling in $x$ : given that the theory of $\S 2$ does not distinguish the streamwise and azimuthal directions, spacefilling structures are required in the latter direction also. Yet evidence to the contrary abounds, namely that long structures only appear in the $x$-direction, induced by the large strain rate. Even then, it is generally accepted that quasi-streamwise vortices are confined to the near-wall region below that in which self-similarity might be expected. A further requirement is that the self-similar motion arises as the result of a hierarchy of non-interacting eddies so that the spectral transfer of energy (the dissipation rate at high Reynolds numbers) is local, as required by the local-equilibrium hypothesis. However, such a condition is inconsistent with the contribution to production by large-scale, supposedly inactive, motion evident in figure 1 which makes the energy processes non-local in both physical and wavenumber space. Bradshaw (1967) has suggested that a near-wall energy balance for the inactive motion alone may be expressed as

$$
\text { advection }=\text { diffusion }+ \text { dissipation, }
$$

in which, although there is direct viscous dissipation arising from the low-wavenumber motion, there is no production. It now seems that (5.2) should be amended to include a production term as well, so that the energy balance is the same as that for the active turbulence. Bradshaw (1967) and Townsend (1976) have analysed the influence of the large scales on the log law: they both suggest that the effective value of $\kappa$ increases with Reynolds number. The foregoing would suggest that, since the outer-region influence increases indefinitely, $\kappa$ will not asymptote to a constant value. However, as the Reynolds number increases, the influence of the larger scales in the horizontal velocity components becomes confined to smaller distances from wall. At $R^{+} \approx 5000$ (when the log law first appears), the effects of inactive motion, most evident at $y^{+} \approx 15$, in fact occupy $O(1 \%)$ of the pipe radius. Therefore any influence of inactive motion on $\kappa$ will be small. 


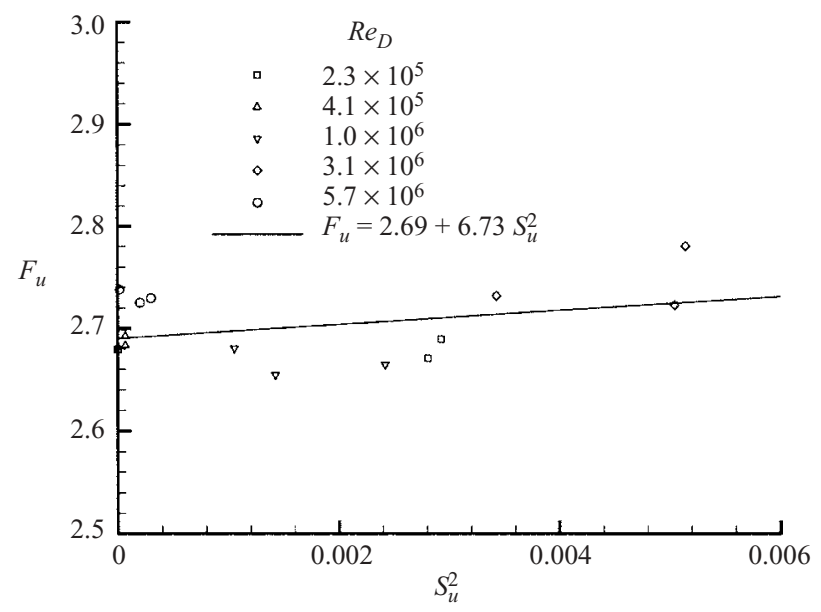

Figure 27. $F_{u}$ as a function of $S_{u}^{2}$.

\subsection{Departures from Gaussian behaviour}

The behaviour of the higher moments in channels has been investigated extensively by Durst \& Jovanović (1995) and Durst et al. (1995). In particular, Jovanović, Durst \& Johansson (1993) have shown that truncated Gram-Charlier series expansions may be used to approximate p.d.f.s in regions in which the second moment reaches a maximum. Representation of higher-order correlations in terms of lower-order correlations is clearly desirable. In view of the fact that the present data show that at high Reynolds numbers there are two maxima (figure 1), the behaviour of the moments and, in particular, their departures from Gaussian over a large range of Reynolds numbers, is especially revealing.

Jovanović, Durst \& Johansson (1993) show that a Gaussian distribution may only be obtained near such a maximum if

$$
\begin{gathered}
S_{j}^{2} \simeq Z Z_{j}^{(1)}\left(F_{j}-3\right), \\
\left(10 S_{j}-S S_{j}\right)^{2} \simeq Z Z_{j}^{(2)}\left(15 F_{j}-30-S F_{j}\right),
\end{gathered}
$$

where $Z Z_{j}^{(1)}$ and $Z Z_{j}^{(2)}$ are functions representing the limiting case of a Gaussian (normal) distribution:

$$
\begin{gathered}
Z Z_{j}^{(1)}=\lim _{\substack{S_{j} \rightarrow 0 \\
F_{j} \rightarrow 3}} \frac{S_{j}^{2}}{F_{j}-3}, \\
Z Z_{j}^{(2)}=\lim _{\substack{S_{j} \rightarrow 0, S S_{j} \rightarrow 0 \\
F_{j} \rightarrow 3, S F_{j} \rightarrow 15}} \frac{\left(10 S_{j}-S S_{j}\right)^{2}}{15 F_{j}-30-S F_{j}} .
\end{gathered}
$$

These expressions lead to simpler ones relating higher moments to lower ones. This is potentially a very useful result if it might be shown that p.d.f.s are invariant with both Reynolds number and position in the shear layer. In particular, they suggest a linear relationship between odd moments, $S S_{u}$ and $S_{u}$, and a quadratic relationship between the even moments, $S F_{u}, F_{u}$ and $S_{u}$. The sublayer peak in $\overline{u^{2}}$ is not amenable to this detailed analysis: however, the peak at $y_{p}^{+}$certainly is. Figures 27 and 29 show $F_{u}$ and $S F_{u}$ respectively, both plotted against $S_{u}^{2}$ while figure 28 shows $S S_{u}$ plotted against $S_{u}$ : only the three data points both at and immediately adjacent to $y_{p}^{+}$are used for the 


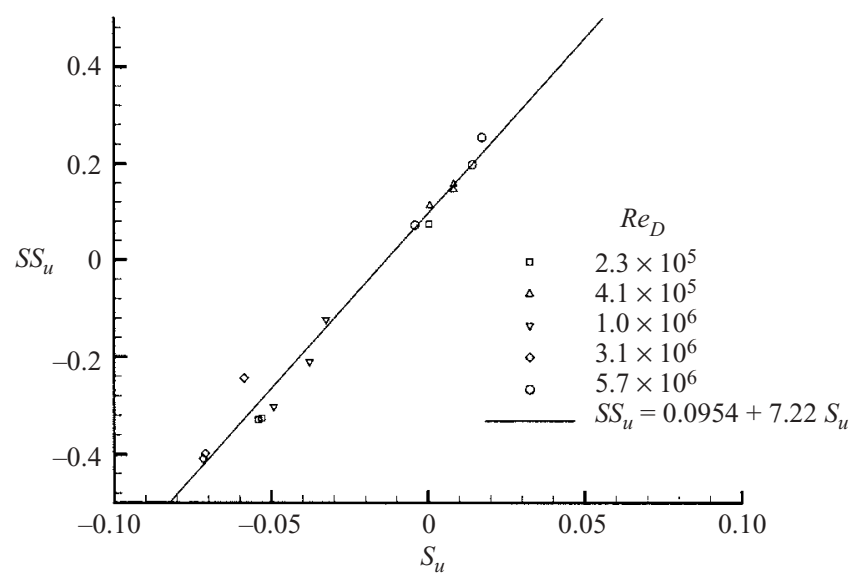

FIGURE 28. $S S_{u}$ as a function of $S_{u}$.

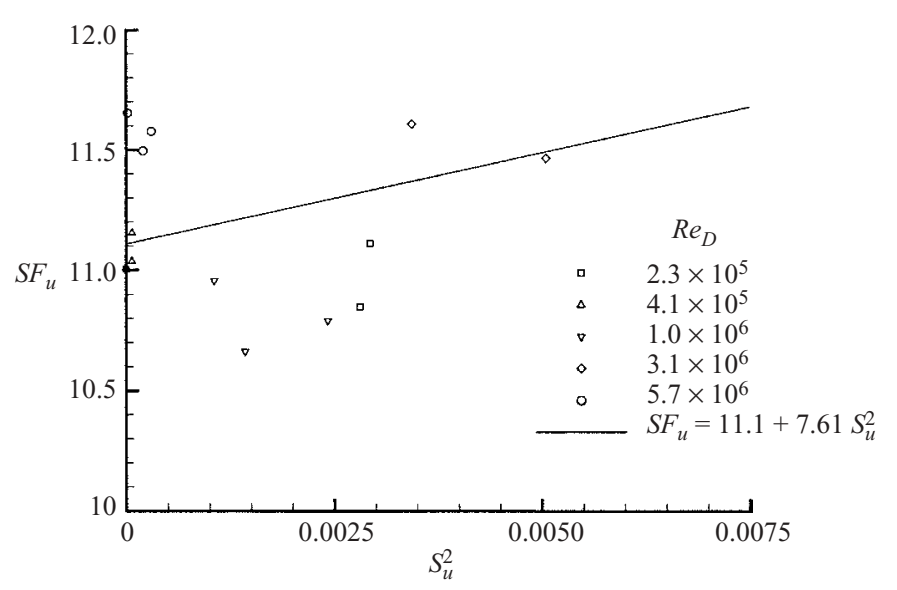

Figure 29. $S F_{u}$ as a function of $S_{u}^{2}$.

Reynolds numbers at which the peak is clearly evident, $2.3 \times 10^{5} \leqslant R e_{D} \leqslant 5.7 \times 10^{6}$. Three points per Reynolds number are used to improve the statistical value of the curve fit. The relationship between the odd moments appears to be close to linear while that between the even moments and $S_{u}^{2}$ is not: figure 30 suggests that the relationship between $S F_{u}$ and $F_{u}$ is the most linear of all. In summary, it appears that the relationships between the higher moments are too complicated to be represented by these simple expressions. In addition, the constants are such that $S F_{u}, S S_{u}$, and $F_{u}$ have significantly non-Gaussian values as $S_{u} \rightarrow 0$. The p.d.f.s of figures 7 and 8 and their moments also show that there are significant variations with both position and Reynolds number.

\subsection{Nature of the peak in $\overline{u^{2}}$ at $y_{p}^{+}$}

The foregoing, and in particular the result of figure 1 which shows two maxima, confirms the principal ideas of so-called inactive motion. The maximum at $y^{+} \approx 15$ is Reynolds-number dependent while its position is only weakly so. Further discussion of this near-wall peak is not possible owing to the poor resolution at higher Reynolds numbers. This is not the case for the outer peak at $y_{p}^{+}$. Using the momentum equation 


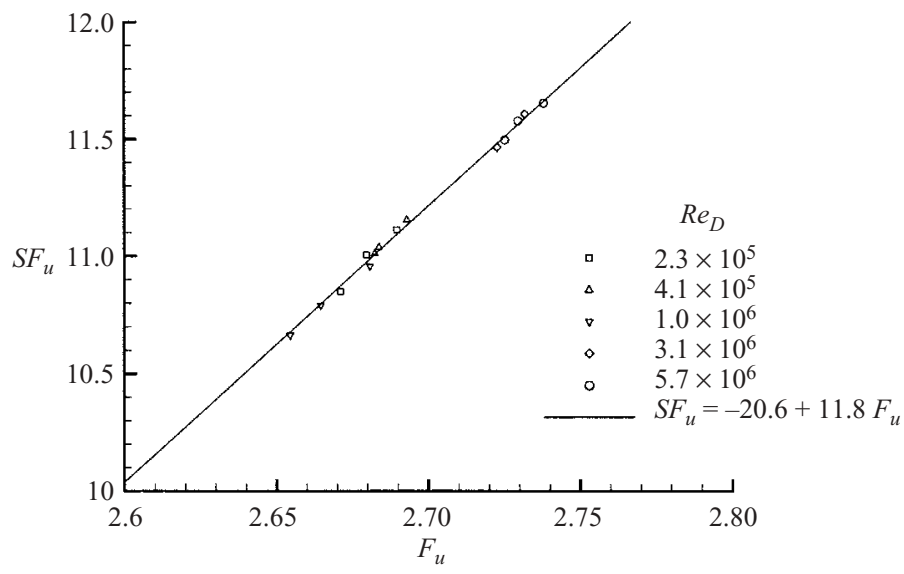

FIGURE 30. $S F_{u}$ as a function of $F_{u}$.

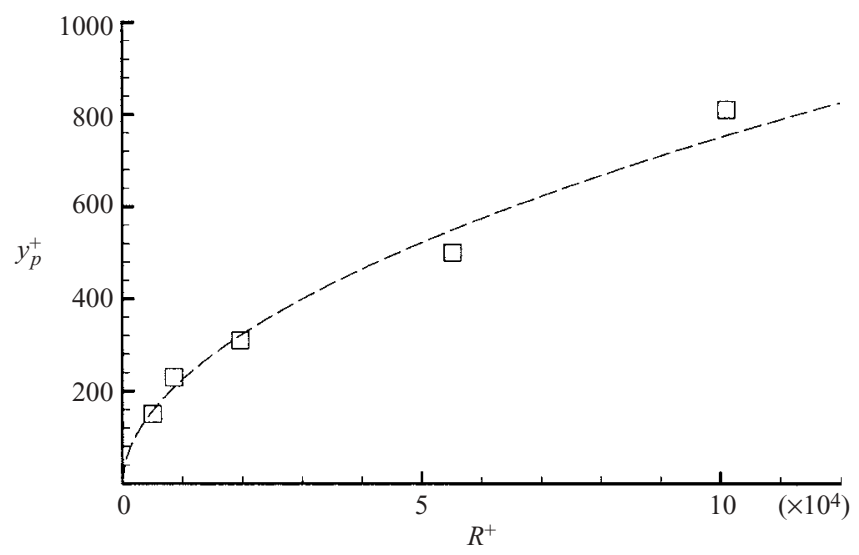

FIGURE 31. Variation of $y_{p}^{+}$with $R^{+}:-----, 1.8\left(R^{+}\right)^{0.52}$.

and assuming the validity of the log law at quite small values of $y^{+}$, the locus of the peak shear stress is given by $\kappa^{-0.5}\left(R^{+}\right)^{0.5}$. Using the values of $\kappa$ given either by Zagarola \& Smits (1998) or by McKeon et al. (2004) gives $1.5\left(R^{+}\right)^{0.5}$ while Sreenivasan (1988), using data from a variety of sources, suggests that the locus is given by $2.0\left(R^{+}\right)^{0.5}$. Figure 31 shows the locus of the maximum in $\overline{u^{2}}, y_{p}^{+}=1.8\left(R^{+}\right)^{0.52}$. Therefore, the mechanism for production of energy at $y^{+} \approx 15$ and of shear stress close to $y_{p}^{+}$are not the same and these differences may be traced back to the different ways in which the wall affects the wall-parallel and wall-normal velocity components. While the outer peak in $\overline{u^{2}}$ closely follows that of $\overline{u v}$, a change in constant would suggest that the $u v$-bearing scales are not necessarily the same as those bearing the turbulence kinetic energy (dominated by $\overline{u^{2}}$ ). Such an interpretation is again consistent with the distinction between active and inactive motion. At $R e_{D}=5.7 \times 10^{6}, y_{p}^{+}=810$ where the wavenumber limit to the spatial resolution is given by $2 \pi y / l=12$. Reference to figure 3 suggests that this estimate of $y_{p}^{+}$is only slightly affected by the limit of spatial resolution.

By analogy with a transitional critical layer, Sreenivasan (1988) has suggested that a 'critical layer' in wall turbulent flow might be a useful model with a vortex sheet 
located at the maximum in Reynolds shear stress. The occurrence of a viscous critical layer where the Reynolds stresses reach a maximum at singularities in the frictionless stability equation leads to an obvious analogue of the peak in Reynolds shear stress in wall turbulent flow at very high Reynolds number. This has implications both for furthering our understanding of inner/outer interactions of wall turbulence as well as for their control.

It is not until the highest Reynolds number that $y_{p}^{+}$reaches the lower limit to the range in which the mean velocity approaches complete similarity (Zagarola \& Smits 1998; McKeon et al. 2004). Using the power law given by Zagarola \& Smits (1998), the mean velocity at $y_{p}^{+}$is given by

$$
U_{p}^{+}=9.43\left(R^{+}\right)^{0.071},
$$

and is more or less a constant fraction of $U_{c l}^{+}$for the range of data given in figure 31, with $0.63 \leqslant U_{p} / U_{c l} \leqslant 0.65$ only (Sreenivasan 1988 finds 0.65 ). Such a result would be expected for a transitional critical layer. Smith \& Bodonyi (1982) have suggested that a lengthscale or thickness of a nonlinear critical layer as a fraction of the pipe diameter is proportional to $\left(R^{+}\right)^{-1 / 6}$ while the classical result (e.g. Stuart 1971) leads to a thickness proportional to $\left(R^{+}\right)^{-1 / 3}$. Unfortunately, while the width of the peak at $y_{p}^{+}$does appear to decrease as the Reynolds number increases, the data of figure 1 cannot be taken as conclusive in this regard. A characteristic velocity fluctuation for the transitional critical layer is $\epsilon U_{0}$ where $U_{0}$ is a characteristic speed, which for our analogy to the turbulent layer is $u_{\tau} . \epsilon$ is the (small) amplitude of disturbance. While the details depend on the nature of the analysis of the transitional critical layer, we take $\epsilon \propto\left(R^{+}\right)^{-1 / 6}$ (Smith \& Bodonyi 1982), in which case the turbulent 'critical layer' has a velocity scale proportional to $u_{\tau}\left(R^{+}\right)^{-1 / 6}$. However, it is clear from figure 1 that the peak at $y_{p}^{+}$increases with Reynolds number. Further work is obviously required.

\section{Conclusions}

We have examined Reynolds-number effects on the second and higher moments of the streamwise velocity component using Townsend's distinction between active and inactive motion. Such an analysis enables the direct effects of viscosity on the near-wall motion to be distinguished from those of the outer-region large eddies. Near-wall maxima in $\overline{u^{2}}$ are Reynolds-number dependent; $\overline{u^{2}}$ does not scale with inner variables and this has to be interpreted as the influence of the outer-layer eddies. The peak at $y^{+} \approx 15$ coincides with that of the production of turbulence kinetic energy and therefore the description of this influence of outer-layer motion as 'inactive' is inappropriate. In addition to so-called inactive motion increasing with Reynolds number, we have shown that it also increases with decreasing wall distance, down to the viscous sublayer, in accord with Townsend's later observations. Statistics show no simple scalings with either inner or outer variables in the same range of $y$ : thus there is no overlap region, a result that is consistent with the above interpretation. Moreover, they also show significant departures from Gaussian over the whole layer, these becoming large both near the wall and the centre line. A comparison of the present outer-region statistics and those of den Toonder \& Nieuwstadt (1997) scaled with both $u_{\tau}$ and $U_{c l}-\bar{U}$ shows that the latter is a better velocity scale, as long as the ranges $R e_{D}<6 \times 10^{4}$ and $R e_{D}>7 \times 10^{4}$ are distinguished. Noting that, even just for the present data, such a conclusion involves at least three sets of independent measurements, it would appear to be a valid one not explainable by anomalies in the data. The change in scaling behaviour appearing in, say, figure 24 , occurs at 
$R e_{D} \approx 6 \times 10^{4}$ and may be traced to the change in $U_{c l}^{+}-\bar{U}^{+}$(which occurs in the range $6 \times 10^{4}<R e_{D}<2 \times 10^{5}$, see McKeon et al. 2004) and the associated changes in the scaling of the first moment. Note also that the second peak in $\bar{u}^{+}$appears only for $R e_{D}>2 \times 10^{5}$. Does this mean that there might be associated structural changes of the form already referred to (perhaps related to hairpin vortex packets described, for example, by Christensen \& Adrian 2001) that might be attributable to a secondary instability? Given the quite low Reynolds numbers involved, such a question might soon be answerable by direct simulation as well as experiments.

The support of ONR under Grant Nos. N00014-98-1-0525 and N00014-99-1-0340 is gratefully acknowledged. J.F. M. is indebted to the Engineering and Physical Sciences Research Council (grants GR/M64536/01 and GR/R48193/01), the Royal Academy of Engineering (England), and the Leverhulme Trust (grant F/07058/H) for financial support.

\section{REFERENCES}

Antonia, R. A. \& Kim, J. 1994 Low-Reynolds-number effects on near-wall turbulence. J. Fluid Mech. 276, 61-80.

Blackwelder, R. F. \& ECKelmanN, H. 1979 The spanwise structure of the bursting phenomenon. J. Fluid Mech. 94, 577-594.

Bradshaw, P. 1967 'Inactive' motion and pressure fluctuations in turbulent boundary layers. J. Fluid Mech. 30, 241-258.

Champagne, F. H., Sleicher, C. A. \& Wehrmann, O. H. 1967 Turbulence measurements with inclined hot wires part 1 . heat transfer experiments with inclined hot wire. J. Fluid Mech. 28, $153-175$.

Christensen, K. T. \& Adrian, R. J. 2001 Statistical evidence of hairpin vortex packets in wall turbulence. J. Fluid Mech. 431, 433-443.

Corrsin, S. 1963 Turbulence: Experimental methods. In Handbuch der Physik, vol. 8, pp. 524-590. Springer.

Dean, R. B. \& Bradshaw, P. 1976 Measurements of interacting turbulent shear layers in a duct. J. Fluid Mech. 78, 641-676.

DeGraAfF, D. B. \& EATON, J. K. 2000 Reynolds-number scaling of the flat-plate turbulent boundary layer. J. Fluid Mech. 422, 319-346.

Dinavahi, S. P. G., Breuer, K. S. \& Sirovich, L. 1995 Universality of probability density functions in turbulent channel flow. Phys. Fluids 7, 1122-1129.

Durst, F. \& Jovanović, J. 1995 Investigations of Reynolds-averaged turbulence quantities. Proc. $R$. Soc. Lond. A 451, 105-120.

Durst, F., Jovanović, J. \& Sender, J. 1995 LDA measurements in the near-wall region of a turbulent pipe flow. J. Fluid Mech. 295, 305-335.

Eggels, J. G. M., Unger, F., Weiss, M. H., Westerweel, J., Adrian, R. J., Friedrich, R. \& Nieuwstadt, F. T. M. 1994 Fully developed turbulent pipe flow: a comparison between direct numerical simulation and experiment. J. Fluid Mech. 268, 175-209.

Fernholz, H. H. \& Finley, P. J. 1996 The incompressible zero-pressure-gradient turbulent boundary layer: an assessment of the data. Prog. Aerospace Sci. 32, 245-311.

Fontaine, A. A. \& Deutsch, S. 1995 Three component, time-resolved velocity statistics in the wall region of a turbulent pipe flow. Exps. Fluids 18, 168-173.

FREYMUTH, P. 1979 Engineering estimation of heat conduction loss in constant temperature thermal sensors. TSI Q. 5, 3-8.

HinZE, J. O. 1975 Turbulence, 2nd edn. McGraw-Hill.

Högström, U., Hunt, J. C. R. \& Smedman, A.-S. 2002 Theory and measurements for turbulence spectra and variances in the atmospheric neutral surface layer. Boundary-Layer Met. 103, $101-124$. 
Hunt, J. C. R. \& Carlotti, P. 2001 Statistical structure at the wall of the high Reynolds number turbulent boundary layer. Flow, Turbulence Combust. 66, 453-475.

Hunt, J. C. R. \& Morrison, J. F. 2000 Eddy structure in turbulent boundary layers. Eur. J. Mech. B/Fluids 19, 673-694.

JimÉNEZ, J. 1998 The largest scales of turbulent wall flows. In Annual Research Briefs, pp. 137-154. Center for Turbulence Research.

Jiménez, J., Del Alámo, J. C. \& Flores, O. 2004 The large-scale dynamics of near-wall turbulence. J. Fluid Mech. 505, 179-199.

JimÉneZ, J., Flores, O. \& García-Villalba, M. 2002 Organization of autonomous wall turbulence. In Advances in Turbulence IX (ed. I. P. Castro, P. E. Hancock \& T. G. Thomas), pp. 824-828. Barcelona: CIMNE.

JimÉnez, J. \& Pinelli, A. 1999 The autonomous cycle of near-wall turbulence. J. Fluid Mech. 389, 335-359.

Jones, M. B., Marusic, I. \& Perry, A. E. 2001 Evolution and structure of sink-flow turbulent boundary layers. J. Fluid Mech. 428, 1-27.

Jovanović, J., Durst, F. \& Johansson, T. G. 1993 Statistical analysis of the dynamic equations for higher-order moments in turbulent wall-bounded flows. Phys. Fluids A 5, 2886-2900.

KAder, B. A. \& Yaglom, A. M. 1991 Spectra and correlation functions of surface layer atmospheric turbulence in unstable thermal stratification. In Turbulence and Coherent Structures (ed. O. Métais \& M. Lesieur). Kluwer.

Katul, G. \& ChiU, C.-R. 1998 A theoretical and experimental investigation of energycontaining scales in the dynamic sublayer of boundary-layer flows. Boundary-Layer Met. 86, 279-312.

Kim, K. C. \& Adrian, R. J. 1999 Very large-scale motion in the outer layer. Phys Fluids. 11, 417-422.

KLEWICKI, J. C. \& FALCO, R. E. 1990 On accurately measuring statistics associated with small-scale structure in turbulent boundary layers using hot-wire probes. J. Fluid Mech. 219, 119-142.

LAUfER, J. 1954 The structure of turbulence in fully developed pipe flow. NACA Tech. Rep. 1174.

Li, J. D., McKeon, B. J., Jiang, W., Morrison, J. F. \& Smits, A. J. 2004 The response of hot wires in high Reynolds-number turbulent pipe flow. Meas. Sci. Technol. 15, 1-10.

Ligrani, P. M. \& Bradshaw, P. 1987 Spatial resolution and measurement of turbulence in the viscous sublayer using subminiature hot-wire probes. Exps. Fluids 5, 407-417.

Long, R. R. \& Chen, T.-C. 1981 Experimental evidence for the existence of the 'mesolayer' in turbulent systems. J. Fluid Mech. 105, 19-59.

Lumley, J. L. 1970 Stochastic Tools in Turbulence. Academic.

Marusic, I. \& Kunkel, G. J. 2003 Streamwise turbulence intensity formulation for flat-plate boundary layers. Phys Fluids. 15, 2461-2464.

Marusic, I. \& KUNKel, G. J. 2004 Turbulence intensity similarity laws for turbulent boundary layers. In IUTAM Symposium on Reynolds Number Scaling in Turbulent Flow (ed. A. J. Smits), pp. 17-22. Kluwer.

Marusic, I. \& Perry, A. E. 1995 A wall-wake model for the turbulence structure of boundary layers. Part 2. Further experimental support. J. Fluid Mech. 298, 389-407.

Marusic, I., Uddin, A. K. M. \& Perry, A. E. 1997 Similarity law for the streamwise turbulence intensity in zero- pressure-gradient turbulent boundary layers. Phys. Fluids 9, 3718-3726.

McKeon, B. J., Li, J., Jiang, W., Morrison, J. F. \& Smits, A. J. 2004 Further observations on the mean velocity in fully-developed pipe flow. J. Fluid Mech. 501, 135-147.

Metzger, M. M. \& Klewicki, J. C. 2001 A comparative study of near-wall turbulence in high and low Reynolds number boundary layers. Phys. Fluids 13, 692-701.

Metzger, M. M., Klewicki, J. C., Bradshaw, K. L. \& Sadr, R. 2001 Scaling the near-wall axial turbulent stress in the zero pressure gradient boundary layer. Phys. Fluids 13, 1819-1821.

Millikan, C. M. 1938 A critical discussion of turbulent flows in channels and circular tube. Proc. 5th Intl Congr. Appl. Mech., pp. 386-392. Wiley.

Mochizuki, S. \& NieuwstadT, F. T. M. 1996 Reynolds-number-dependence of the maximum in the streamwise velocity fluctuations in wall turbulence. Exps. Fluids 21, 218-226.

Morris, S. C. \& Foss, J. F. 2003 Transient thermal response of a hot-wire anemometer. Meas. Sci. Technol. 14, 251-259. 
Morrison, J. F., Jiang, W., McKeon, B. J. \& Smits, A. J. 2001 Reynolds-number dependence of streamwise velocity fluctuations in turbulent pipe flow. In Turbulence and Shear Flow Phenomena, TSFP-2, pp. 43-48. Stockholm: Universitetsservice US AB.

Morrison, J. F., Jiang, W., McKeon, B. J. \& Smits, A. J. $2002 a$ Reynolds-number dependence of streamwise velocity fluctuations in turbulent pipe flow. AIAA Paper 2002-0574.

Morrison, J. F., Jiang, W., McKeon, B. J. \& Smits, A. J. $2002 b$ Reynolds-number dependence of streamwise velocity spectra in turbulent pipe flow. Phys. Rev. Lett. 88, 214501.

Morrison, J. F., Subramanian, C. S. \& Bradshaw, P. 1992 Bursts and the law of the wall in turbulent boundary layers. J. Fluid Mech. 241, 75-108.

Nieuwstadt, F. T. M. \& Bradshaw, P. 1997 Similarities and differences of turbulent boundarylayer, pipe and channel flow. In Boundary-Layer Separation in Aircraft Aerodynamics (ed. R. A. W. M. Henkes \& P. G. Bakker). Delft University Press.

Nikora, V. 1999 Origin of the '-1' spectral law in wall-bounded turbulence. Phys. Rev. Lett. 83, 734-736.

Perry, A. E. \& Abell, C. J. 1975 Scaling laws for pipe-flow turbulence. J. Fluid Mech. 67, 257-271.

Perry, A. E. \& Abell, C. J. 1977 Asymptotic similarity of turbulence structures in smooth- and rough-walled pipes. J. Fluid Mech. 79, 785-799.

Perry, A. E., Henbest, S. \& Chong, M. S. 1986 A theoretical and experimental study of wall turbulence. J. Fluid Mech. 165, 163-199.

Perry, A. E. \& LI, J. D. 1990 Experimental support for the attached-eddy hypothesis in zeropressure-gradient turbulent boundary layers. J. Fluid Mech. 218, 405-438.

Perry, A. E. \& Marusic, I. 1995 A wall-wake model for the turbulence structure of boundary layers. Part 1. Extension of the attached eddy hypothesis. J. Fluid Mech. 298, 361-388.

RotTA, J. C. 1962 Turbulent boundary layers in incompressible flow. Prog. Aeronaut. Sci. 2, 1-220.

SANDBORN, V. A. 1955 Experimental evaluation of momentum terms in turbulent pipe flow. NACA Tech. Note 3266.

Smith, F. T. \& Bodonyi, R. J. 1982 Amplitude-dependent neutral modes in the Hagen-Poiseuille flow through a circular pipe. Proc. R. Soc. Lond. A 384, 463-489.

SreEnivasan, K. R. 1988 A unified view on the origin and morphology of the turbulent boundary layer structure. In Turbulence Management and Relaminarization (ed. H. W. Liepmann \& R. Narasimha), pp. 37-62. Springer.

StUart, J. T. 1971 Non-linear stability theory. Annu. Rev. Fluid Mech. 3, 347-370.

TCHEN, C. M. 1953 On the spectrum of energy in turbulent shear flow. J. Res. Natl Bureau Stand. 50, 51-62.

Den Toonder, J. M. J. \& Nieuwstadt, F. T. M. 1997 Reynolds number effects in a turbulent pipe flow for low to moderate Re. Phys. Fluids 9, 3398-3409.

Townsend, A. A. 1956 The Structure of Turbulent Shear Flow, 1st edn. Cambridge University Press.

Townsend, A. A. 1961 Equilibrium layers and wall turbulence. J. Fluid Mech. 11, 97-120.

Townsend, A. A. 1976 The Structure of Turbulent Shear Flow, 2nd edn. Cambridge University Press.

Tsuji, Y. \& NakamuRa, I. 1999 Probability density function in the log-law region of low reynolds number turbulent boundary layer. Phys. Fluids 11, 647-658.

Wei, T. \& Willmarth, W. W. 1989 Reynolds number effects on the structure of a turbulent channel flow. J. Fluid Mech. 204, 57-95.

Westbury, P. S. \& MorRison, J. F. 1995 Bursts and low-Reynolds-number effects in turbulent boundary layers. In Advances in Turbulence $V$ (ed. R. Benzi), pp. 549-553. Kluwer.

Wosnik, M., Castillo, W. L. \& George, K. 2000 A theory for turbulent pipe and channel flows. J. Fluid Mech. 421, 115-145.

Zagarola, M. V., Perry, A. E. \& Smits, A. J. 1997 Log laws or power laws: The scaling in the overlap region. Phys. Fluids 9, 2094-2100.

Zagarola, M. V. \& Smits, A. J. 1998 Mean-flow scaling of turbulent pipe flow. J. Fluid Mech. 373, 33-79. 